\title{
A FINITE ELEMENT, MULTIRESOLUTION VISCOSITY METHOD FOR HYPERBOLIC CONSERVATION LAWS*
}

\author{
MARCUS CALHOUN-LOPEZ ${ }^{\dagger}$ AND MAX D. GUNZBURGER ${ }^{\ddagger}$
}

\begin{abstract}
It is well known that the classic Galerkin finite element method is unstable when applied to hyperbolic conservation laws such as the Euler equations for compressible flow. It is also well known that naively adding artificial diffusion to the equations stabilizes the method but sacrifices too much accuracy to be of any practical use. An elegant approach, referred to as spectral viscosity methods, has been developed for spectral methods in which one adds diffusion only to the high-frequency modes of the solution, the result being that stabilization is effected without sacrificing accuracy. We extend this idea into the finite element framework by using hierarchical finite element functions as a multifrequency basis. The result is a new finite element method for solving hyperbolic conservation laws in which artificial diffusion can be applied selectively only to the high-frequency modes of the approximation. As for spectral viscosity methods, this results in stability without compromising accuracy. In the context of a one-dimensional scalar hyperbolic conservation law, we prove the convergence of approximate solutions, obtained using the new method, to the entropy solution of the conservation law. To illustrate the method, the results of a computational experiment for a one-dimensional hyperbolic conservation law are provided.
\end{abstract}

Key words. hyperbolic conservation laws, finite element methods, multiresolution viscosity, hierarchical basis functions

AMS subject classifications. $65 \mathrm{~N} 60,35 \mathrm{~L} 65$

DOI. $10.1137 /$ S0036142904439380

1. Introduction. We consider a new finite element method, based on hierarchical basis functions and a scale-dependent artificial viscosity, for hyperbolic conservation laws. With respect to a given triangulation of a domain, standard nodal basis functions are all of the same scale; i.e., their support is roughly equal. In contrast, hierarchical basis functions can be clustered into groups such that basis functions within a particular group are of a different scale from those of the other groups. The multiscale nature of the hierarchical basis functions allows for the selective addition of viscosity only at the smallest scales, very much in the spirit of spectral viscosity methods. It is hoped that such flexibility will be sufficient for stabilizing discrete Galerkin finite element approximations while, at the same time, results in more accurate approximations with respect to both convergence rates in regions where the solution is smooth and the sharpness of the resolution of discontinuities in the solution.

This paper is a first step at verifying that finite element methods based on hierarchical basis functions and a scale-dependent artificial viscosity do indeed fulfill the promise mentioned in the previous paragraph. We provide some background

${ }^{*}$ Received by the editors January 5, 2004; accepted for publication (in revised form) October 18, 2004; published electronically December 15, 2005.

http://www.siam.org/journals/sinum/43-5/43938.html

$\dagger$ Mathematics Department, University of Maryland, College Park, MD 20742-4015 (mcalhoun@ math.umd.edu). The research of this author was supported in part by National Science Foundation grant DMS-0240049 and by the U.S. Department of Energy, Office of Energy Research and performed at Sandia National Laboratories, a multiprogram laboratory operated by Sandia Corporation, a Lockheed-Martin Company, for the United States Department of Energy's National Nuclear Security Administration under contract DE-AC-94AL85000.

${ }^{\ddagger}$ School of Computational Science, Florida State University, Tallahassee, FL 32306-4120 (gunzburg@csit.fsu.edu). The research of this author was supported in part by National Science Foundation grant DMS-0308845 and by CSRI, Sandia National Laboratories, contract 18407. 
material and then describe the new method. We then analyze it for the case of a one-dimensional, periodic, scalar hyperbolic conservation law, showing that, under appropriate hypotheses, the approximate solution converges to the entropy solution of the conservation law. We then provide a simple example of the use of the method. Several issues concerning the efficient implementation of the new method as well as the results of more extensive computational testing are provided in $[3,4]$.

1.1. Hyperbolic conservation laws. Let $\Omega \subseteq \mathbb{R}^{d}$ be a bounded domain. A general system of conservation laws has the form

$$
\frac{\partial \mathbf{q}}{\partial t}+\sum_{j=1}^{d} \frac{\partial}{\partial x_{j}} \mathbf{f}_{j}(\mathbf{q})=\mathbf{0} \quad \text { in } \Omega \times(0, \infty) \quad \text { and } \quad \mathbf{q}(\cdot, 0)=\mathbf{g} \quad \text { in } \Omega
$$

along with appropriate boundary conditions. Here, $\mathbf{q}: \Omega \times[0, \infty) \rightarrow \mathbb{R}^{p}$ denotes the vector-valued conserved variable, $\mathbf{f}_{j}: \mathbb{R}^{p} \rightarrow \mathbb{R}^{p}, j=1, \ldots, d$, denote the $d$ flux functions, and $\mathbf{g}: \Omega \rightarrow \mathbb{R}^{p}$ denotes the given initial data. For $\widetilde{\mathbf{q}} \in \mathbb{R}^{p}$, let $\mathbf{A}_{j}(\widetilde{\mathbf{q}})$ : $\mathbb{R}^{p} \rightarrow \mathbb{R}^{p \times p}$ denote the $p \times p$ Jacobian matrix of $\mathbf{f}_{j}$, i.e., $\mathbf{A}_{j}(\widetilde{\mathbf{q}})=\frac{\partial \mathbf{f}_{j}}{\partial \mathbf{q}}(\widetilde{\mathbf{q}})$. The system (1.1) is hyperbolic if, for all solutions $\mathbf{q}$, any linear combination of $\left\{\mathbf{A}_{j}(\mathbf{q})\right\}_{j=1}^{d}$ has real eigenvalues with eigenvectors that span $\mathbb{R}^{p}$. The system (1.1) is strictly hyperbolic if the eigenvalues are distinct. See, e.g., $[8,10]$ for details.

The system (1.1) does not, in general, have a classical solution because of the spontaneous formation of discontinuities. Instead, one must look for a solution $\mathbf{q} \in$ $L^{\infty}\left(\Omega \times(0, \infty) ; \mathbb{R}^{p}\right)$ which satisfies (1.1) in the distributional sense:

$$
\int_{0}^{\infty} \int_{\Omega}\left[\mathbf{q} \cdot \frac{\partial \phi}{\partial t}+\sum_{j=1}^{d} \mathbf{f}_{j}(\mathbf{q}) \cdot \frac{\partial \phi}{\partial x_{j}}\right] d \Omega d t+\int_{\Omega} \mathbf{g} \cdot \boldsymbol{\phi}(\cdot, 0) d \Omega=0
$$

for all test functions $\phi \in C_{0}^{\infty}\left(\Omega \times[0, \infty) ; \mathbb{R}^{p}\right)$. It is clear that for a smooth enough solution, (1.1) and (1.2) are equivalent.

In the presence of discontinuities, solutions of the system (1.1) or of the weak formulation (1.2) are not uniquely determined. Additional conditions must be imposed to determine the unique, physically relevant solution. The second law of thermodynamics tells us that the entropy of the system should not decrease; satisfying this requirement suffices to allow one to obtain the unique, physically relevant entropy solution.

Let $\Phi,\left\{\Psi_{j}\right\}_{j=1}^{d}: \mathbb{R}^{p} \rightarrow \mathbb{R}$ be smooth functions; for (1.1), $\Phi$ is an entropy with entropy fluxes $\left\{\Psi_{j}\right\}_{j=1}^{d}$ if $\Phi$ is convex and $\boldsymbol{\nabla}_{\mathbf{q}} \Phi^{T} \frac{\partial \mathbf{f}_{j}}{\partial \mathbf{q}}=\nabla_{\mathbf{q}} \Psi_{j}$ in $\mathbb{R}^{p}$ for $1 \leq j \leq d$. A simple calculation states that if $\mathbf{q}$ is a smooth solution to (1.1), then $\Phi(\mathbf{q})$ satisfies a scalar conservation law with flux $\Psi(\mathbf{q})$ :

$$
\frac{\partial}{\partial t} \Phi(\mathbf{q})+\sum_{j=1}^{d} \frac{\partial}{\partial x_{j}} \Psi_{j}(\mathbf{q})=0 \text { in } \mathbb{R}^{d} \times(0, \infty) .
$$

In some instances, $\Phi$ can be interpreted as the negative of the physical entropy, so (1.3) says that if $\mathbf{u}$ is a smooth solution, then $\Phi \circ \mathbf{q}$ satisfies a conservation law with flux functions $\left\{\Psi_{j} \circ \mathbf{q}\right\}_{j=1}^{d}$.

For solutions with discontinuities, we impose the entropy condition on $\mathbf{q}$ that requires the physical entropy to be nondecreasing:

$$
\frac{\partial}{\partial t} \Phi(\mathbf{q})+\sum_{j=1}^{d} \frac{\partial}{\partial x_{j}} \Psi_{j}(\mathbf{q}) \leq 0
$$


for every entropy function $\Phi$ with entropy fluxes $\{\Psi\}_{j=1}^{d} ;(1.4)$ is an inequality in the distributional sense:

$$
\int_{0}^{\infty} \int_{\Omega}\left[\Phi(\mathbf{q}) \frac{\partial \phi}{\partial t}+\sum_{j=1}^{d} \Psi_{j}(\mathbf{q}) \frac{\partial \phi}{\partial x_{j}}\right] d \Omega d t \geq 0 \forall \phi \in C_{0}^{\infty}(\Omega \times(0, \infty)), \phi \geq 0 .
$$

In (1.1), viscous effects are ignored. For the class of phenomena that are modeled with hyperbolic conservation laws, viscous effects are generally small, but they are present and play a role when sharp gradients (such as shocks) of the solution are present. An alternate and equivalent means of characterizing the unique, physically relevant solution of (1.1) is to let $\mathbf{q}=\lim _{\varepsilon \rightarrow 0} \mathbf{q}^{\varepsilon}$ a.e., where $\mathbf{q}^{\varepsilon}: \Omega \times[0, \infty) \rightarrow \mathbb{R}^{p}$ is the solution of the perturbed equation

$$
\frac{\partial \mathbf{q}^{\varepsilon}}{\partial t}+\sum_{j=1}^{d} \frac{\partial}{\partial x_{j}} \mathbf{f}_{j}\left(\mathbf{q}^{\varepsilon}\right)-\varepsilon \Delta \mathbf{q}^{\varepsilon}=\mathbf{0} \text { in } \Omega \times(0, \infty) \quad \text { and } \quad \mathbf{q}^{\varepsilon}(\cdot, 0)=\mathbf{g} \text { in } \Omega
$$

along with boundary conditions. In other words, the entropy solution is the limit of the viscous solution as the viscosity goes to zero.

1.2. Numerical methods for hyperbolic conservation laws. Direct discretizations of (1.1) lead to unstable approximations. The most obvious stabilization approach is to instead discretize the perturbed system (1.6) but, as is well known, this leads to severe smearing of discontinuities and to low accuracy even in regions in which the solution is smooth. Of course, there have been many methods proposed for determining improved stabilized approximation solutions of hyperbolic conservation laws; see, e.g., $[6,10,13,17,18,24,27]$.

Finite difference (FD) methods are the oldest of the numerical methods, so many variations have been developed. Many successful strategies for solving hyperbolic conservation laws were originally developed in the FD framework then adapted to other methods. However, FD schemes tend to have difficulties with complex geometries, satisfying prescribed boundary conditions, and rigorous analyses. In fluid dynamics, complex geometries are common, and, as shown in [6], poor approximation of boundary conditions can severely affect a numerical method.

Finite volume (FV) methods inherently capture many of the important aspects of conservation laws; FV methods are locally conservative. Information is propagated along the characteristic curves, at least approximately. FV methods use unstructured grids, so they can handle complex geometries. High-order schemes, however, are difficult to attain.

Finite element (FE) methods are well suited to handle complex geometries and prescribed boundary conditions. Formally high-order schemes can be defined by simply increasing the degree of the approximating polynomials used. The price paid is a large increase in the number of unknowns. In discontinuous Galerkin (DG) methods (see, e.g., [6]), no continuity restrictions are placed on the approximating solution, which results in several advantages. DG methods are easy to parallelize; adaptive strategies are relatively easy to implement; and the mass matrix is block diagonal, so explicit time-stepping schemes are possible. The lack of continuity of solutions, however, is also the cause of the biggest drawback of DG methods: The number of unknowns is drastically increased compared to, say, nodal finite element methods and other types of methods. The shock capturing streamline diffusion method adds a 
diffusion term to the conservation law, but unlike (1.6), diffusion is added in different amounts in the direction of the characteristic curves (streamline diffusion) and its normal direction (crosswind diffusion). Streamline diffusion is added everywhere, while crosswind diffusion is added only near discontinuities. To determine characteristic curves, space-time elements must be used, which increases the number of unknowns and results in an implicit time scheme. See [13,14,23].

Spectral methods, including the spectral viscosity (SV) method, provide another class of methods. Since incorporating the ideas from the SV method into the FE framework is the subject of this paper, we discuss SV methods in a little more detail in section 1.2.1.

The distinctions between the various methods are not always sharp. Some FV and FE schemes can be written into an equivalent FD formulation. As noted in [6], some FV methods can be considered to be special types of DG methods. Furthermore, there are other methods, e.g., kinetic methods [22], for hyperbolic conservation laws that do not fall within the classes just mentioned.

1.2.1. Spectral viscosity methods. In [24], SV methods were introduced as a scheme to obtain approximate solutions of the periodic Burgers equation using Fourier spectral basis functions. The theory was further refined and extended in a series of papers $[5,9,12,19,20,25,26]$. Of particular importance to us are $[12,19]$, in which Legendre polynomials are used. The variational formulation of the Legendre $\mathrm{SV}$ method is closest to our FE formulation.

We present the most basic SV method, which uses Fourier spectral basis functions. Using standard notation from Fourier spectral methods, we define

$$
u_{N}=P_{N} u(x, t), \quad P_{N} u=\sum_{|k| \leq N} \widehat{u}_{k}(t) e^{i k \pi x}, \quad \widehat{u}_{k}(t)=\frac{1}{2} \int_{-1}^{+1} u(x, t) e^{-i k \pi x} d \Omega .
$$

We seek $u_{N}$ such that

$$
\frac{\partial u_{N}}{\partial t}+\frac{\partial}{\partial x}\left(P_{N} \frac{u_{N}^{2}}{2}\right)=\varepsilon \frac{\partial}{\partial x}\left(Q_{N} \frac{\partial u_{N}}{\partial x}\right) .
$$

$Q_{N}$ denotes the spectral viscosity operator defined as a convolution with the viscosity kernel, $Q_{N}(x)$, so that

$$
Q_{N} \frac{\partial u_{N}}{\partial x}=Q_{N}(x) * \frac{\partial u_{N}(x, t)}{\partial x} \quad \text { and } \quad Q_{N}(x)=\sum_{|k| \leq N} \widehat{Q}_{k} e^{i k \pi x} .
$$

We choose $0 \leq \widehat{Q}_{k} \leq 1$ and $\widehat{Q}_{k}=0$ for small $|k|$. It is easy to see the effect of $Q_{N}$ if we write the diffusion term in Fourier space:

$$
\varepsilon \frac{\partial}{\partial x}\left(Q_{N} \frac{\partial u_{N}}{\partial x}\right)=-\varepsilon \sum_{|k| \leq N}\left(k^{2} \pi^{2} \widehat{Q}_{k} \widehat{u}_{k} e^{i k \pi x}\right) .
$$

Since $\widehat{Q}_{k}=0$ for all but large $|k|, Q_{N}$ dampens or eliminates the low frequency modes of $u_{N}$ in the diffusion term. So, we see that the SV diffusion term is a compromise between not adding diffusion, which leads to instability, and adding full diffusion, which limits the convergence rate and smears out discontinuities in the solution. Ideally, one would like to add diffusion only in the vicinity of a discontinuity. However, the global nature of the basis functions does not allow for an adaptive viscosity kernel. 
The SV solution $u_{N}$ does not converge to the exact solution $u$ at the optimal rate because of the poor convergence of $P_{N} u$. $P_{N} u$ is limited to first-order convergence in smooth regions and has $O(1)$ Gibbs oscillations near a discontinuity. Post-processing $u_{N}$ recovers spectral convergence. The post-processing scheme can be enhanced by knowing the locations of discontinuities, as in [9]. Because of the global nature of spectral basis functions, this edge detection task is not trivial.

1.3. Hierarchical finite element basis functions. The usual (nodal) basis functions used in FE methods all have the same frequency. In order to have multifrequency basis functions, we use hierarchical basis functions. In the elliptic partial differential equation setting, an early analysis of hierarchical basis functions, especially in one dimension, is given in [31]. For two dimensions, see [29]. A good overview of hierarchical basis functions can be found in [30].

First consider a polygonal domain $\Omega$. Let $\mathcal{T}_{0}$ be a coarse grid approximation of $\Omega$. The $n$ th-level triangulation $\mathcal{T}_{n}$ is obtained by subdividing the elements of $\mathcal{T}_{n-1}$. Let $S^{N}$ be the space of continuous functions which are polynomials of degree $p$ on the elements of $\mathcal{T}_{N}$. Let $\mathcal{N}_{N} \subseteq \bar{\Omega}$ be the nodes of the elements of $\mathcal{T}_{N}$. The nodal basis functions of $S^{N}$ are defined by $\phi_{i} \in S^{N}$ such that $\phi_{i}\left(x_{j}\right)=\delta_{i j}$ for all $x_{j} \in \mathcal{N}_{N}$. It is well known that $S^{N}=\operatorname{span}\left\{\phi_{i}\right\}_{i}$. The use of nodal bases leads to many nice numerical properties, such as sparse matrices and the local assembly of matrices. However, we cannot use the nodal bases for our purposes because, as we noted earlier, the elements of $\left\{\phi_{i}\right\}_{i}$ all have the same frequency.

Let $\mathcal{N}_{n}$ denote the nodes of the $n$ th-level triangulation, $\mathcal{T}_{n}, \mathcal{S}^{n}$ denote the corresponding finite element space, and $B^{n}$ denote the nodal basis of $\mathcal{S}^{n}$. The hierarchical basis functions are defined by

$$
\psi_{n, i} \in B^{n} \quad \text { such that } \quad \psi_{n, i}\left(x_{j}\right)=0 \quad \forall x_{j} \in \mathcal{N}_{n-1} .
$$

For $0 \leq n \leq N,\left\{\psi_{n, i}\right\}_{n, i} \subseteq S^{N}$ is a linearly independent set with the same dimension as $S^{N}$, so $S^{N}=\operatorname{span}\left\{\psi_{n, i}\right\}_{n, i}$. See Figure 1 for a comparison of the nodal and hierarchical bases for linear elements in one dimension. As can be seen from the figure, $\psi_{n, i}$ is a low frequency function for small $n$ and a high frequency function for large $n$.

The strategy just outlined works for polynomials of any degree. For example, the first column of Figure 2 consists of quadratic hierarchical basis functions. An alternate strategy is to use linear hierarchical basis functions for $n<N$, as in the second column of Figure 2.

In order to determine $\mathcal{T}_{n+1}$ from $\mathcal{T}_{n}$, we must decide, for a given $T \in \mathcal{T}_{n}$, how many subelements to divide $T$ into. For linear and quadratic rectangular-type elements in $\mathbb{R}^{d}$, the natural choice is $2^{d}$ subelements. For cubic elements, the natural choice is $3^{d}$ since the vertices of an element will then be a subset of the vertices of its parent. Here, we limit our attention to linear and quadratic basis functions.

For domains with curved boundaries, the situation is more complicated. Let $\Omega$ be our potentially complicated domain. One strategy is to use the hierarchical decomposition of some polygonal domain $\Omega^{\prime}$ such that $\Omega \subseteq \Omega^{\prime}$, as in [15]. Another strategy is to try and impose a hierarchical structure on an unstructured mesh, as in [1]. The hierarchical structure could also be imposed on the mapping of $\Omega$ to a polygonal domain.

Several important properties of hierarchical finite element basis functions and several issues that arise in efficient implementations of finite element methods based on these kinds of bases are discussed in $[3,4]$. 
Linear Hierarchical Basis Functions level 0: 2 functions

level 1: 1 function

level 2: 2 functions

level 3: 4 functions
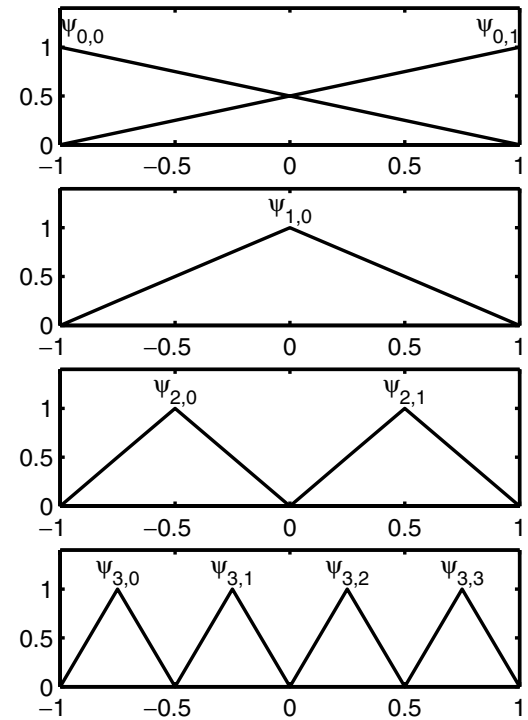

Standard Linear Basis Functions 9 functions

FIG. 1. Hierarchical (left) and nodal (right) linear basis functions spanning the same ninedimensional finite element space.

2. Finite element multiresolution viscosity method. Assume we have a hierarchical sequence of partitions $\left\{\mathcal{T}_{n}\right\}_{n=0}^{N}$ of the open bounded set $\Omega \subseteq \mathbb{R}^{d}$. Let $\mathbf{S}^{N}$ be the space of continuous vector-valued functions whose components are in $S^{N}$. We seek an approximate solution to the hyperbolic conservation law (1.1). The finite element multiresolution viscosity method is defined as follows: seek $\mathbf{q}^{N} \in \mathbf{S}^{N}$ such that

$$
\begin{aligned}
& \frac{d}{d t} \int_{\Omega} \mathbf{q}^{N} \cdot \mathbf{v} d \Omega+\sum_{j=1}^{d} \int_{\Omega} \frac{\partial}{\partial x_{j}} \mathbf{f}_{j}\left(\mathbf{q}^{N}\right) \cdot \mathbf{v} d \Omega \\
& \quad+\varepsilon_{N} \sum_{i=1}^{p} \sum_{j, k=1}^{d} \int_{\Omega} \frac{\partial}{\partial x_{j}}\left(Q_{N}^{j, k} q_{i}^{N}\right) \frac{\partial v_{i}}{\partial x_{k}} d \Omega \\
& \quad-\varepsilon_{N} \sum_{i=1}^{p} \sum_{j, k=1}^{d} \int_{\partial \Omega} \frac{\partial}{\partial x_{j}}\left(Q_{N}^{j, k} q_{i}^{N}\right) v_{i} \widehat{n}_{k} d s=0 \quad \forall \mathbf{v} \in \mathbf{S}^{N},
\end{aligned}
$$

where $\widehat{\mathbf{n}}$ is the unit normal to the boundary $\partial \Omega$ of $\Omega$. As in the SV method, $Q_{N}^{j, k}$ is chosen to dampen or eliminate the low frequency modes of a function:

$$
\left\{\begin{array}{l}
Q_{N}^{j, k}: S^{N} \rightarrow S^{N}, \quad \sum_{n=0}^{N} \sum_{i} \beta_{n, i} \psi_{n, i} \mapsto \sum_{n=0}^{N} \sum_{i} Q_{N ; n, i}^{j, k} \beta_{n, i} \psi_{n, i}, \\
0 \leq Q_{N ; n, i}^{j, k} \leq 1, \quad \text { and } \quad Q_{N ; n, i}^{j, k}= \begin{cases}0 & \text { for small } n(n \text { near } 0), \\
1 & \text { for large } n(n \text { near } N) .\end{cases}
\end{array}\right.
$$



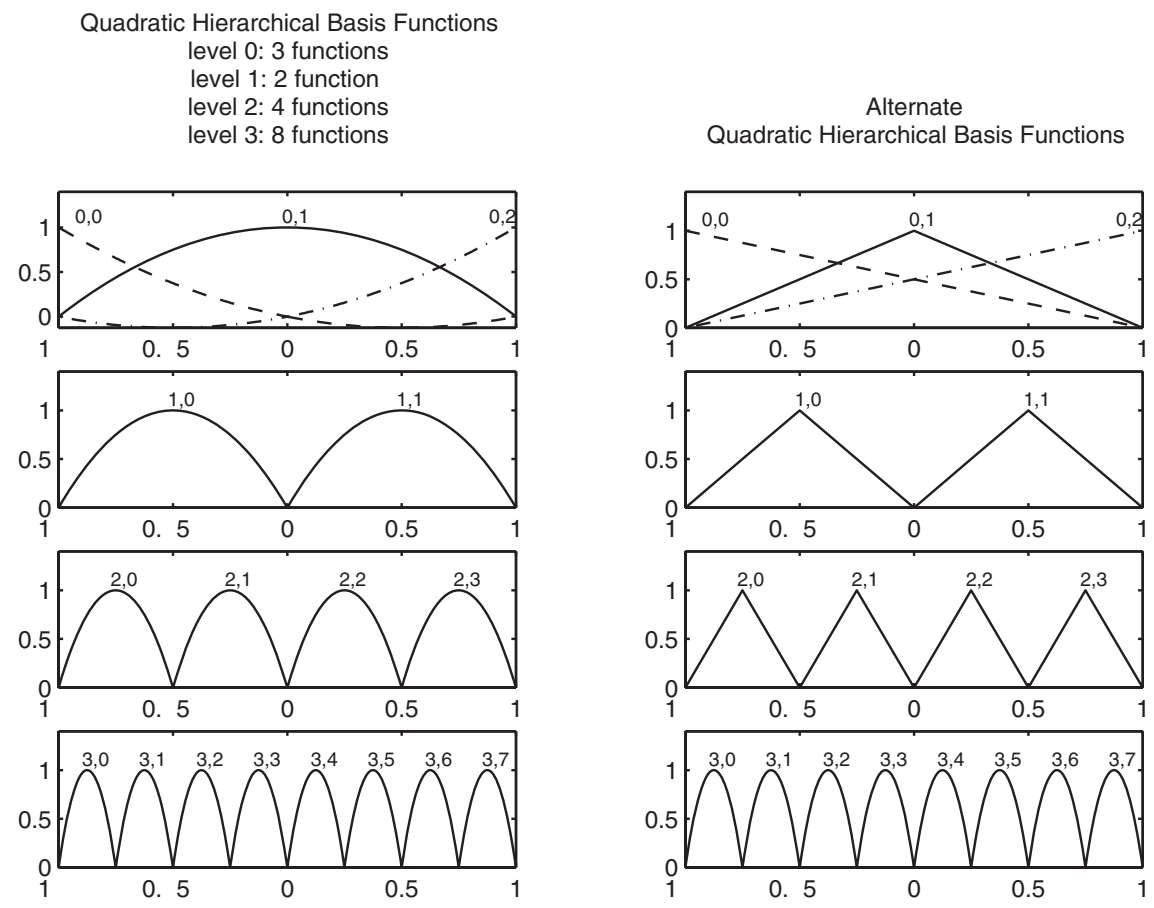

FIG. 2. Two sets (left and right) of quadratic hierarchical basis functions spanning the same 17-dimensional, quadratic finite element space.

To account for boundary conditions imposed along with (1.1), a subspace of $S_{N}$ might need to be used (for essential boundary conditions) or the boundary integral in (2.1) might be reduced to one over part of $\partial \Omega$ (for natural boundary conditions).

Equation (2.1) is a weak formulation of the modified system

$$
\frac{\partial \mathbf{q}}{\partial t}+\sum_{j=1}^{d} \frac{\partial}{\partial x_{j}} \mathbf{f}_{j}(\mathbf{q})-\varepsilon_{N} \sum_{j, k=1}^{d} \frac{\partial^{2}}{\partial x_{j} \partial x_{k}}\left(Q_{N}^{j, k} \mathbf{q}\right)=\mathbf{0},
$$

where $\left[Q_{N}^{j, k} \mathbf{q}\right]_{i}=Q_{N}^{j, k} q_{i}$ for $1 \leq i \leq p$. The dependence of $Q_{N}^{j, k}$ on both $j$ and $k$ allows for the flexibility of introducing directional bias in the diffusion which can result in reduced crosswind diffusion. As in the streamline diffusion method, this would probably require the use of entropy variables. Here, we simplify our formulation by using an isotropic diffusion term, $Q_{N}$, such that

$$
Q_{N ; n, i}^{j, k}=Q_{N ; n, i} \delta_{j, k} .
$$

Then, (2.1) and (2.3), respectively, reduce to

$$
\begin{gathered}
\frac{d}{d t} \int_{\Omega} \mathbf{q} \cdot \mathbf{v} d \Omega+\sum_{j=1}^{d} \int_{\Omega} \frac{\partial}{\partial x_{j}} \mathbf{f}_{j}(\mathbf{q}) \cdot \mathbf{v} d \Omega+\varepsilon_{N} \int_{\Omega} \boldsymbol{\nabla}\left(Q_{N} \mathbf{q}\right): \nabla \mathbf{v} d \Omega \\
-\varepsilon_{N} \int_{\partial \Omega} \frac{\partial}{\partial n}\left(Q_{N} \mathbf{q}\right) \cdot \mathbf{v} d s=0 \quad \forall \mathbf{v} \in \mathbf{S}^{N}
\end{gathered}
$$


and

$$
\frac{\partial \mathbf{q}}{\partial t}+\sum_{j=1}^{d} \frac{\partial}{\partial x_{j}} \mathbf{f}_{j}(\mathbf{q})-\varepsilon_{N} \Delta\left(Q_{N} \mathbf{q}\right)=\mathbf{0}
$$

After choosing a time discretization technique, (2.4) is equivalent to a nonlinear system of equations that may be solved, e.g., by Newton's method. The relevant Jacobian matrix has the form $J^{H}=\widetilde{J}^{H}+K^{H} Q$, where $\widetilde{J}^{H}$ is the Jacobian of the time dependent and flux terms, $K^{H}$ is the Laplacian stiffness matrix, and $Q$ is a diagonal matrix whose nonzero elements are $\left\{Q_{N ; n, i}\right\}$. For ease of presentation, we have ignored the boundary term. Note that for an explicit time integration method, the Jacobian matrix reduces to the mass matrix.

The solution of the discrete equations resulting from our hierarchical finite element discretization may be implemented using matrices arising from the corresponding nodal basis. See $[3,4]$ for details. Here, we merely observe that the Jacobian matrix $J^{H}$ and residual vector $\vec{R}^{H}$ may be expressed, respectively, in terms of their nodal basis counterparts $J^{D}$ and $\vec{R}^{D}$ through the relations $J^{H}=S^{T}\left(\widetilde{J}^{D} S+K^{D} S Q\right)$ and $\vec{R}^{H}=S^{T} \vec{R}^{D}$, where $S$ is the change of basis matrix such that $\vec{X}^{D}=S \vec{X}^{H}$. The determination of $\left(J^{H}\right)^{-1} \vec{R}^{H}=\left(\widetilde{J}^{D} S+K^{D} S Q\right)^{-1} \vec{R}^{D}$ by an iterative solver then requires the calculation of the matrix-vector multiplication $\left(\widetilde{J}^{D} S+K^{D} S Q\right) \vec{X}$ and possibly $\left(\widetilde{J}^{D} S+K^{D} S Q\right)^{T} \vec{X}$ that only involve the nodal matrices $\widetilde{J}^{D}$ and $K^{D}$ and the transfer matrix $S$. Compared to $K^{D}$ and $\widetilde{J}^{D}, S$ is not sparse, so one does not want to explicitly construct $S$. So, making the iterative linear solvers efficient requires being able to calculate $S \vec{X}$ and $S^{T} \vec{X}$ quickly. Algorithms for this purpose can be found in $[3,4]$.

2.1. Advantages of hierarchical bases. We use hierarchical bases (instead of nodal bases) because of their multifrequency property. Nodal bases, however, have important computational advantages such as producing matrices that are much more sparse and that can be locally assembled. However, as just discussed, one can retain most of the advantages of the nodal bases. One assembles and stores all of the system matrices as nodal basis matrices and uses the transfer matrix $S$ in an iterative linear solver; $S$ does not even have to be stored.

In the SV method, there is only one function, with global support, at a given frequency. In the hierarchical $\mathrm{FE}$ formulation, there are many functions, with local support, at a given frequency. Compared to SV methods, the hierarchical FE formulation offers two advantages: Diffusion can be added locally, and edge detection is trivial. For large values of $n$, the hierarchical basis function $\psi_{n, i}$ has local support, so $Q_{N: n, i}^{j, k}$ only has a local effect. One can therefore add more diffusion near a discontinuity and less or no diffusion in smooth regions. This should improve the accuracy of the method. As we are about to see, the size of $\left|\beta_{n, i}\right|$ (where $\beta_{n, i}$ is the coefficient of $\psi_{n, i}$ in the hierarchical basis expansion of a function; see (2.2)) can be used to determine if the support of $\psi_{n, i}$ resides in a smooth region or is near a discontinuity.

2.1.1. Edge detection. Using hierarchical bases, edge detection is a trivial task. Near a discontinuity, the high frequency hierarchical coefficients are of order one. In smooth regions, they shrink exponentially. Figure 3 illustrates a hierarchical decomposition of a piecewise smooth function containing a discontinuity. One can easily determine the location of discontinuities by looking at the magnitude of the coefficients of the high frequency basis functions. 

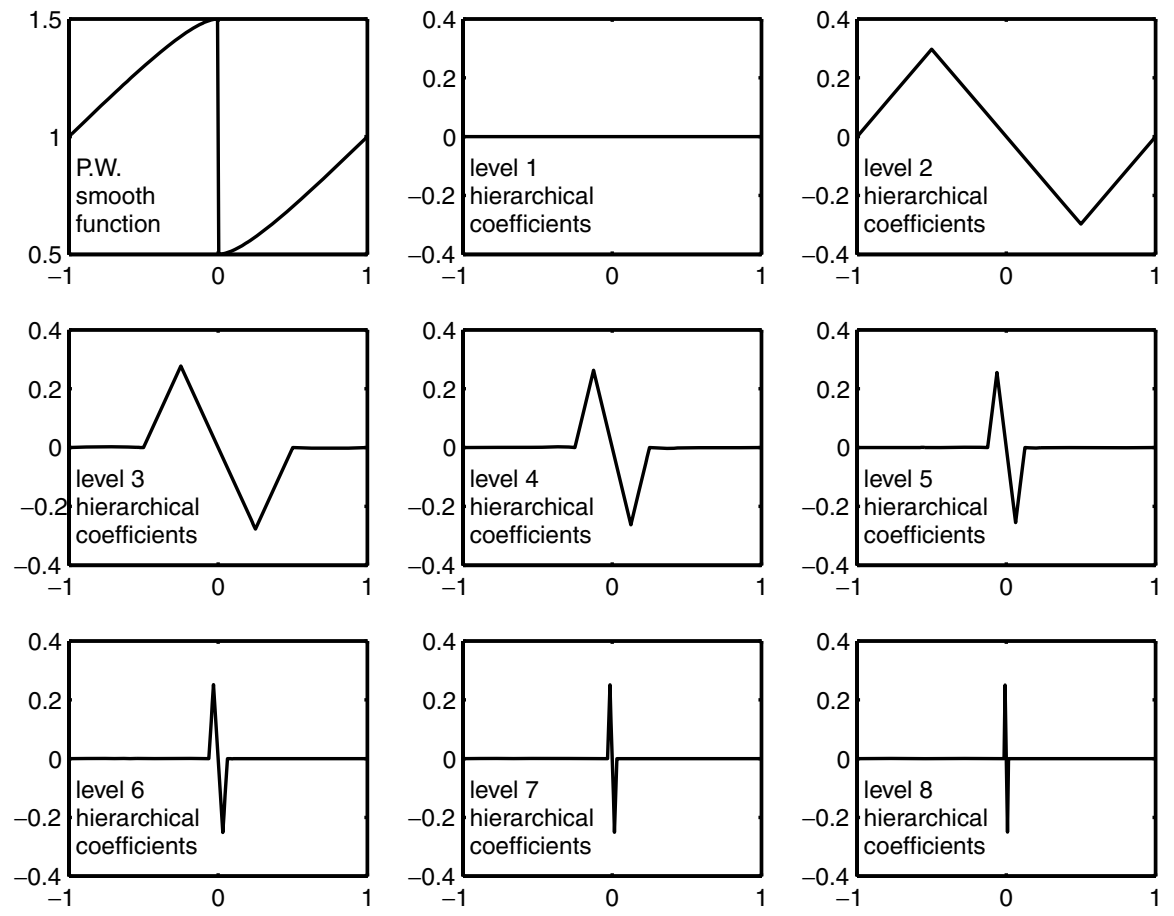

FIG. 3. Hierarchical decomposition of a piecewise smooth function with a discontinuity.

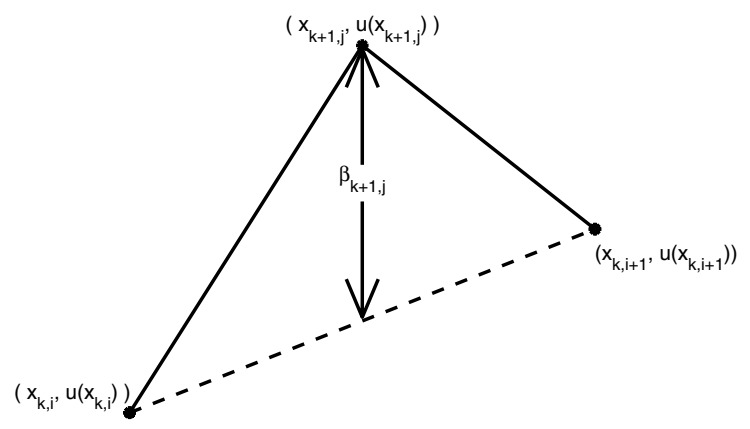

FIG. 4. Determination of a linear hierarchical coefficient from function values.

Edge detection for piecewise linear polynomials. Let us examine the behavior of the hierarchical coefficients for linear hierarchical basis functions. As one can see in Figure $4, \beta_{k+1, j}$ can be calculated from the value of the function $u$ at $x_{k+1, j}$ and at the node points of the parent cell. For a uniform partition, the cell size at level $k$ is $\Delta x_{k}=|\Omega| 2^{-k}$. Thus, $x_{k+1, j}-x_{k, i}=x_{k, i+1}-x_{k+1, j}=\Delta x_{k+1}$ and

$$
\beta_{k+1, j}=\frac{u\left(x_{k+1, j}\right)-u\left(x_{k, i}\right)}{2}-\frac{u\left(x_{k, i+1}\right)-u\left(x_{k+1, j}\right)}{2} .
$$

Let $T_{k, i}=\left(x_{k, i}, x_{k, i+1}\right)$.

Assume that $u$ is discontinuous and has a discontinuity in $T_{k, i}$. Then, at least one of the two terms in (2.5) will have a relatively large value so that $\left|\beta_{k+1, j}\right|$ will be 
of the same order as the jump of $u$, independent of $k$.

Now, assume that $u$ is continuously differentiable, i.e., $u \in C^{1}\left(\overline{T_{k, i}}\right)$. Then, since (2.5) can be written as

$$
\beta_{k+1, j}=\frac{|\Omega|}{4} 2^{-k}\left(\frac{u\left(x_{k+1, j}\right)-u\left(x_{k, i}\right)}{x_{k+1, j}-x_{k, i}}-\frac{u\left(x_{k, i+1}\right)-u\left(x_{k+1, j}\right)}{x_{k, i+1}-x_{k+1, j}}\right),
$$

the mean value theorem yields that

$$
\beta_{k+1, j}=\frac{|\Omega|}{4} 2^{-k}\left[u^{\prime}\left(\widetilde{x}_{1}\right)-u^{\prime}\left(\widetilde{x}_{2}\right)\right]
$$

for some $\widetilde{x}_{1}, \widetilde{x}_{2} \in T_{k, i}$. Therefore, $\beta_{k+1, j}$ is of order $2^{-k}$, i.e.,

$$
\left|\beta_{k+1, j}\right| \leq|\Omega|\left\|u^{\prime}\right\|_{L^{\infty}\left(T_{k, i}\right)} 2^{-k-1}
$$

so that it decays exponentially with $k$.

If $u$ is twice continuously differentiable, i.e., $u \in C^{2}\left(\overline{T_{k, i}}\right)$, the mean value theorem applied to (2.6) yields that

$$
\beta_{k+1, j}=-\frac{|\Omega|}{4} 2^{-k}\left(\widetilde{x}_{2}-\widetilde{x}_{1}\right) u^{\prime \prime}(\widetilde{x})
$$

for some $\widetilde{x} \in T_{k, i}$. Therefore, $\beta_{k+1, j}$ is of order $4^{-k}$, i.e.,

$$
\left|\beta_{k+1, j}\right| \leq \frac{|\Omega|}{4}\left|\widetilde{x}_{2}-\widetilde{x}_{1}\right|\left|u^{\prime \prime}(\widetilde{x})\right| 2^{-k} \leq|\Omega|^{2}\left\|u^{\prime \prime}\right\|_{L^{\infty}\left(T_{k, i}\right)} 4^{-k-1}
$$

so that it again decays exponentially with $k$.

Similar results can be obtained for quadratic hierarchical basis function; see [3,4].

3. Convergence to entropy solutions for one-dimensional scalar conservation laws. In this section, we prove that the solution of the hierarchical finite element discretization introduced in section 2 converges to the entropy solution of the one-dimensional, periodic Burgers equation. We will make use of the method of compensated compactness; in particular, we will use the div-curl lemma $[7,21,28]$ and Murat's lemma $[7,13,19,21,28]$. The broad outlines of the proof follow that of [24].

3.1. The periodic, one-dimensional Burgers equation. Let $\Omega=(a, b)$ be an open bounded interval and let $\Omega_{T}=(a, b) \times(0, T)$ for some finite time interval $(0, T)$. We seek a finite element $(\mathrm{FE})$ approximation to $u(x, t)$, the entropy solution of the periodic hyperbolic conservation law:

$$
\begin{aligned}
& \frac{\partial u}{\partial t}+\frac{\partial}{\partial x}\left(\frac{u^{2}}{2}\right)=0 \quad \text { in } \Omega_{T}, \\
& u(a, t)=u(b, t) \quad \text { in }(0, T), \quad \text { and } \quad u(x, 0)=g \quad \text { in }(a, b),
\end{aligned}
$$

along with the entropy condition

$$
\frac{\partial}{\partial t}\left(\frac{u^{2}}{2}\right)+\frac{\partial}{\partial x}\left(\frac{u^{3}}{3}\right) \leq 0 \quad \text { in } \Omega_{T}
$$

where (3.1) and (3.3) hold in the distributional sense. We will assume the $g \in H^{1}(a, b)$ and that $g$ is space-periodic. The entropy solution of Burgers' equation can be found 
using (3.3) instead of the more general entropy condition (1.4). This greatly simplifies our analysis since we now require an entropy-type inequality for one entropy/entropy flux pair, $\left(\frac{u^{2}}{2}, \frac{u^{3}}{3}\right)$, instead of all of them. A weak form of the problem (3.1) is given by

$$
\int_{\Omega_{T}}\left(\varphi \frac{\partial u}{\partial t}+\varphi \frac{\partial}{\partial x}\left(\frac{u^{2}}{2}\right)\right) d x d t=0 \quad \forall \varphi \in C_{0}^{\infty}\left(\Omega_{T}\right)
$$

Correspondingly, (3.3) can be expressed in the weak form

$$
\int_{\Omega_{T}}\left(\varphi \frac{\partial}{\partial t}\left(\frac{u^{2}}{2}\right)+\varphi \frac{\partial}{\partial x}\left(\frac{u^{3}}{3}\right)\right) d x d t \leq 0 \quad \forall \varphi \in C_{0}^{\infty}\left(\Omega_{T}\right), \varphi \geq 0 .
$$

3.1.1. The hierarchical finite element discretization. To formulate the FE approximation, we need some notation. Let $\mathcal{T}_{0}=(a, b) ; \mathcal{T}_{N}$ is obtained by subdividing the elements of $\mathcal{T}_{N-1}$ into $M$ distinct elements. Let $|b-a| h_{N}$ be the maximal diameter of the elements of $\mathcal{T}_{N}$. Since we assume that the partition is quasi-uniform, there exists a positive constant $\nu$ such that $M^{-N} \leq h_{N} \leq \nu M^{-N}$ for all $N$.

Let $\left\{\psi_{k, i}\right\}_{k, i}$ be a hierarchical basis of $S_{p}^{N}$. Let us define $Q_{N}: S_{p}^{N} \rightarrow S_{p}^{N}$ as a damping operator $Q_{N} u_{N}=\sum_{k, i}\left(Q_{k, i} \beta_{k, i} \psi_{k, i}\right)$ for $u=\sum_{k, i}\left(\beta_{k, i} \psi_{k, i}\right) \in S_{p}^{N}$, where $0 \leq Q_{k, i} \leq 1$ and $Q_{k, i}=1$ for $k>m_{H}$. Thus, $Q_{N}$ dampens (or eliminates) the low frequencies of a function while keeping the high frequencies above the level $m_{H}$. Occasionally, when the level of a basis function is unimportant, we will switch to the less cumbersome notation $\left\{\psi_{i}\right\}_{i}$ and $\left\{Q_{i}\right\}_{i}$ for the basis functions and damping coefficients, respectively.

We will also use the following convention: $C$ will denote any positive constant which depends on known quantities and is independent of any indexing variables.

Let $g_{N} \in S_{p}^{N}$ be the interpolant of $g$. The hierarchical finite element approximation of $(3.1)-(3.2)$ is given by the following: seek $u_{N}(x, t)$ with $u_{N}(\cdot, 0)=g_{N}$ such that, for all $v \in S_{p}^{N}$,

$$
\int_{a}^{b}\left[\frac{\partial u_{N}}{\partial t}+\frac{\partial}{\partial x}\left(\frac{u_{N}^{2}}{2}\right)\right] v d x+\varepsilon_{N} \int_{a}^{b}\left[\frac{\partial}{\partial x}\left(Q_{N} u_{N}\right) \frac{\partial v}{\partial x}\right] d x=0 .
$$

3.2. Convergence theorem. We prove the following convergence results for hierarchical finite element approximations of the entropy solution of (3.1) and (3.2).

TheOREM 3.1. Let $\left\{u_{N}\right\}_{N=0}^{\infty}$ denote a sequence of hierarchical finite element approximations determined by (3.6). Assume that $\left\|u_{N}\right\|_{L^{\infty}\left(\Omega_{T}\right)} \leq C$ and assume that

$$
\begin{aligned}
& \varepsilon_{N}, h_{N} \rightarrow 0 \text { as } N \rightarrow \infty \\
& \frac{\varepsilon_{N}}{h_{N}} \geq C \\
& \sqrt{\varepsilon_{N}}\left\|\frac{\partial}{\partial x}\left[\left(I-Q_{N}\right) v_{N}\right]\right\|_{L^{2}(a, b)} \leq C\left\|v_{N}\right\|_{L^{2}(a, b)} \text { for } v_{N} \in\left\{u_{N}, \frac{\partial u_{N}}{\partial t}\right\} \\
& \left\|\frac{d}{d x}\left(Q_{N} g_{N}\right)\right\|_{L^{2}(U)} \leq C\left\|\frac{d g_{N}}{d x}\right\|_{L^{2}(U)} .
\end{aligned}
$$

Then, there exists a subsequence of $\left\{u_{N}\right\}_{N=0}^{\infty}$ that converges strongly in $L^{2}\left(\Omega_{T}\right)$ to a solution $u \in L^{2}\left(\Omega_{T}\right)$ of (3.1) and (3.2). Further, assume that

$$
\frac{\varepsilon_{N}}{h_{N}} \rightarrow \infty \text { as } N \rightarrow \infty \text { and }
$$




$$
\sqrt{\varepsilon_{N}}\left\|\frac{\partial}{\partial x}\left[\left(I-Q_{N}\right) u_{N}\right]\right\|_{L^{2}(a, b)} \rightarrow 0 \text { as } N \rightarrow \infty .
$$

Then the subsequence of $\left\{u_{N}\right\}_{N}^{\infty}$ converges strongly in $L^{2}\left(\Omega_{T}\right)$ to the entropy solution of (3.1) and (3.2), i.e., to the solution of (3.1)-(3.3).

For the moment, we assume that (3.7)-(3.12) hold, and we prove, in sections 3.3 to 3.7 , the theorem. Subsequently, in section 3.8, we will show that these conditions are satisfied in our context.

3.3. Existence of the finite element approximation. The discrete FE equations (3.6) are equivalent to the following: seek $\vec{\alpha}:(0, T) \rightarrow \mathbb{R}^{s}$, where $s=\operatorname{dim} S_{p}^{N}$, such that

$$
\dot{\vec{\alpha}}+M^{-1} \vec{F}(\vec{\alpha})+M^{-1} K Q \vec{\alpha}=\overrightarrow{0},
$$

where $M$ is the mass matrix, $K$ is the stiffness matrix, $Q$ is a diagonal matrix whose elements are $\left\{Q_{i}\right\}$, and $\vec{F}$ is the flux term: $[\vec{F}(\vec{\alpha})]_{i}=\int_{a}^{b} \psi_{i} \frac{1}{2} \frac{\partial}{\partial x}\left(\sum_{j} \alpha_{j} \psi_{j}\right)^{2} d x=$ $\vec{\alpha}^{T} A_{i} \vec{\alpha}$, where $A_{i}$ is the symmetric matrix $\left(A_{i}\right)_{j, k}=\frac{1}{2} \int_{a}^{b} \psi_{i} \frac{\partial}{\partial x}\left(\psi_{j} \psi_{k}\right) d x$. Evidently, the diffusion term is globally Lipschitz continuous. We now show that the flux term is locally Lipschitz continuous. For all $\vec{\alpha}, \vec{\beta} \in \mathbb{R}^{s}$ and all $i$,

$$
\begin{aligned}
\left|[\vec{F}(\vec{\beta})-\vec{F}(\vec{\alpha})]_{i}\right| & =\left|\vec{\beta}^{T} A_{i} \vec{\beta}-\vec{\alpha}^{T} A_{i} \vec{\alpha}\right|=\left|(\vec{\beta}+\vec{\alpha})^{T} A_{i}(\vec{\beta}-\vec{\alpha})\right| \\
& \leq\|\vec{\beta}+\vec{\alpha}\|_{2}\left\|A_{i}\right\|_{2}\|\vec{\beta}-\vec{\alpha}\|_{2} \leq \sqrt{s}\|\vec{\beta}+\vec{\alpha}\|_{2}\left\|A_{i}\right\|_{2}\|\vec{\beta}-\vec{\alpha}\|_{\infty}
\end{aligned}
$$

so that $\|\vec{F}(\vec{\beta})-\vec{F}(\vec{\alpha})\|_{\infty} \leq \sqrt{s}\|\vec{\beta}+\vec{\alpha}\|_{2} \max _{1 \leq i \leq s}\left\|A_{i}\right\|_{2}\|\vec{\beta}-\vec{\alpha}\|_{\infty}$. Since $\left\|A_{i}\right\|_{2}$ and $s$ are independent of $\vec{\alpha}$ and $\vec{\beta}$, the flux term is locally Lipschitz continuous for any $T$.

Lipschitz continuity together with $|\vec{\alpha}(t)|<\infty$, by (3.15) and (3.17), yields that there exists a unique $\vec{C}^{1}[0, T]$ solution of (3.13) or, equivalently, of (3.6).

3.4. Estimates for $\boldsymbol{u}_{N}$. In (3.6), choose $v=u_{N}$; then

$$
\int_{a}^{b}\left[\frac{\partial}{\partial t}\left(\frac{u_{N}^{2}}{2}\right)+\frac{\partial}{\partial x}\left(\frac{u_{N}^{3}}{3}\right)\right] d x+\varepsilon_{N} \int_{a}^{b} \frac{\partial}{\partial x}\left(Q_{N} u_{N}\right) \frac{\partial u_{N}}{\partial x} d x=0 .
$$

Since $u_{N}$ is periodic, $\int_{a}^{b} \frac{\partial}{\partial x}\left(\frac{u_{N}{ }^{3}}{3}\right) d x=0$ so that

$$
\frac{1}{2} \frac{d}{d t}\left\|u_{N}\right\|_{L^{2}(a, b)}^{2}+\varepsilon_{N} \int_{a}^{b} \frac{\partial}{\partial x}\left(Q_{N} u_{N}\right) \frac{\partial u_{N}}{\partial x} d x=0 .
$$

3.4.1. $\boldsymbol{H}^{\mathbf{1}}\left(\boldsymbol{\Omega}_{T}\right)$ estimates for $\boldsymbol{u}_{N}$ for linear polynomials. The elements of the piecewise linear hierarchical basis are orthogonal with respect to the $H^{1}(a, b)$ seminorm. As a result,

$$
\begin{aligned}
& \int_{a}^{b} \frac{\partial}{\partial x}\left(Q_{N} u_{N}\right) \frac{\partial u_{N}}{\partial x} d x=\sum_{i} \sum_{j} Q_{i} \beta_{i} \beta_{j} \int_{a}^{b} \psi_{i}^{\prime} \psi_{j}^{\prime} d x \\
& \quad=\sum_{i} Q_{i} \beta_{i}^{2} \int_{a}^{b}\left(\psi_{i}^{\prime}\right)^{2} d x \geq \sum_{i} Q_{i}^{2} \beta_{i}^{2} \int_{a}^{b}\left(\psi_{i}^{\prime}\right)^{2} d x \\
& \quad=\sum_{i} \sum_{j} Q_{i} Q_{j} \beta_{i} \beta_{j} \int_{a}^{b} \psi_{i}^{\prime} \psi_{j}^{\prime} d x=\left\|\frac{\partial}{\partial x}\left(Q_{N} u_{N}\right)\right\|_{L^{2}(a, b)}^{2} .
\end{aligned}
$$


Integrating (3.14) over time, we obtain

$$
\begin{aligned}
C\|g\|_{L^{2}(a, b)}^{2} & \geq\left\|g_{N}\right\|_{L^{2}(a, b)}^{2}=\left\|u_{N}(\cdot, t)\right\|_{L^{2}(a, b)}^{2}+2 \varepsilon_{N} \int_{0}^{t} \int_{a}^{b} \frac{\partial}{\partial x}\left(Q_{N} u_{N}\right) \frac{\partial u_{N}}{\partial x} d x d t \\
& \geq\left\|u_{N}(\cdot, t)\right\|_{L^{2}(a, b)}^{2}+2 \varepsilon_{N} \int_{0}^{t}\left\|\frac{\partial}{\partial x}\left(Q_{N} u_{N}\right)\right\|_{L^{2}(a, b)}^{2} d t
\end{aligned}
$$

so that

$$
\left\|u_{N}\right\|_{L^{2}\left(\Omega_{T}\right)} \leq C \sqrt{T}\|g\|_{L^{2}(a, b)}, \quad \sqrt{\varepsilon_{N}}\left\|\frac{\partial}{\partial x}\left(Q_{N} u_{N}\right)\right\|_{L^{2}\left(\Omega_{T}\right)} \leq C\|g\|_{L^{2}(a, b)} .
$$

3.4.2. $H^{1}\left(\Omega_{T}\right)$ estimates for $u_{N}$ for quadratic polynomials. The quadratic hierarchical basis functions are not orthogonal, but we can still obtain an estimate similar to (3.15). We now have that

$$
\begin{aligned}
& \int_{a}^{b} \frac{\partial}{\partial x}\left(Q_{N} u_{N}\right) \frac{\partial u_{N}}{\partial x} d x=\int_{a}^{b} \frac{\partial}{\partial x}\left(Q_{N} u_{N}\right) \frac{\partial}{\partial x}\left(Q_{N} u_{N}\right) d x \\
& \quad+\int_{a}^{b} \frac{\partial}{\partial x}\left(Q_{N} u_{N}\right) \frac{\partial}{\partial x}\left[\left(I-Q_{N}\right) u_{N}\right] d x \\
& \geq \int_{a}^{b}\left|\frac{\partial}{\partial x}\left(Q_{N} u_{N}\right)\right|^{2} d x \\
& \quad-\frac{1}{2} \int_{a}^{b}\left|\frac{\partial}{\partial x}\left(Q_{N} u_{N}\right)\right|^{2} d x-\frac{1}{2} \int_{a}^{b}\left|\frac{\partial}{\partial x}\left[\left(I-Q_{N}\right) u_{N}\right]\right|^{2} d x \\
& =\frac{1}{2}\left\|\frac{\partial}{\partial x}\left(Q_{N} u_{N}\right)\right\|_{L^{2}(a, b)}^{2}-\frac{1}{2}\left\|\frac{\partial}{\partial x}\left[\left(I-Q_{N}\right) u_{N}\right]\right\|_{L^{2}(a, b)}^{2} \\
& \geq \frac{1}{2}\left\|\frac{\partial}{\partial x}\left(Q_{N} u_{N}\right)\right\|_{L^{2}(a, b)}^{2}-\frac{C}{2 \varepsilon_{N}}\left\|u_{N}\right\|_{L^{2}(a, b)}^{2} \cdot
\end{aligned}
$$

Substituting this result into (3.14), we obtain

$$
\frac{d}{d t}\left\|u_{N}\right\|_{L^{2}(a, b)}^{2} \leq C\left\|u_{N}\right\|_{L^{2}(a, b)}^{2}-\varepsilon_{N}\left\|\frac{\partial}{\partial x}\left(Q_{N} u_{N}\right)\right\|_{L^{2}(a, b)}^{2} .
$$

We require a nonstandard formulation of the differential form of Gronwall's inequality. A proof is given in [8].

Lemma 3.2. Let $\eta(t)$ be an absolutely continuous function on $[0, T]$ such that for a.e. $t \in[0, T], \eta^{\prime}(t) \leq \phi(t) \eta(t)+\psi(t)$, where $\phi(t)$ and $\psi(t)$ are summable functions on $[0, T]$. Then, $\eta(t) \leq e^{\int_{0}^{t} \phi(r) d r}\left[\eta(0)+\int_{0}^{t} e^{-\int_{0}^{s} \phi(r) d r} \psi(s) d s\right] \forall t \in[0, T]$.

Let us now assume that $\phi=C$ is a positive constant, and $\psi \leq 0$ is never positive. We then have

$$
\begin{aligned}
\eta(t) & \leq e^{C t}\left[\eta(0)+\int_{0}^{t} e^{-C s} \psi(s) d s\right] \\
& \leq e^{C t}\left[\eta(0)+\int_{0}^{t} e^{-C t} \psi(s) d s\right]=e^{C t} \eta(0)+\int_{0}^{t} \psi(s) d s .
\end{aligned}
$$

Using this result with (3.16), we obtain

$$
\left\|u_{N}\right\|_{L^{2}(a, b)}^{2} \leq e^{C t}\left\|g_{N}\right\|_{L^{2}(a, b)}^{2}-\varepsilon_{N} \int_{0}^{t}\left\|\frac{\partial}{\partial x}\left(Q_{N} u_{N}\right)\right\|_{L^{2}(a, b)}^{2} d s .
$$


Since $\left\|g_{N}\right\|_{L^{2}(a, b)} \leq C\|g\|_{L^{2}(a, b)}$,

$$
\left\|u_{N}\right\|_{L^{2(a, b)}}^{2}+\varepsilon_{N} \int_{0}^{t}\left\|\frac{\partial}{\partial x}\left(Q_{N} u_{N}\right)\right\|_{L^{2(a, b)}}^{2} d s \leq C e^{C t}\|g\|_{L^{2(a, b)}}^{2} .
$$

Therefore, we have that

$$
\left\{\begin{array}{l}
\left\|u_{N}\right\|_{L^{2}\left(\Omega_{T}\right)} \leq C \sqrt{e^{C T}-1}\|g\|_{L^{2}(a, b)}, \\
\sqrt{\varepsilon_{N}}\left\|\frac{\partial}{\partial x}\left(Q_{N} u_{N}\right)\right\|_{L^{2}\left(\Omega_{T}\right)} \leq C \sqrt{e^{C T}}\|g\|_{L^{2}(a, b)} .
\end{array}\right.
$$

3.5. Strong convergence of $\left\{\boldsymbol{u}_{N}\right\}$. Let $\mathbf{v}_{N}=\left(\frac{u_{N}^{2}}{2}, u_{N}\right)$ and $\mathbf{w}_{N}=\left(\frac{u_{N}^{2}}{2},-\frac{u_{N}{ }^{3}}{3}\right)$ so that

$\operatorname{div} \mathbf{v}_{N}=\frac{\partial u_{N}}{\partial t}+\frac{\partial}{\partial x}\left(\frac{u_{N}^{2}}{2}\right) \quad$ and $\quad \operatorname{curl} \mathbf{w}_{N}=\frac{\partial}{\partial t}\left(\frac{u_{N}^{2}}{2}\right)+\frac{\partial}{\partial x}\left(\frac{u_{N}^{3}}{3}\right)$.

3.5.1. $L^{2}\left(\boldsymbol{\Omega}_{T}\right)$ bound on $\left\{\operatorname{div} \mathbf{v}_{N}\right\}$. In (3.6), choose $v=\frac{\partial u_{N}}{\partial t}$; then

$$
\begin{aligned}
& \int_{a}^{b}\left|\frac{\partial u_{N}}{\partial t}\right|^{2} d x+\int_{a}^{b} \frac{\partial}{\partial x}\left(\frac{u_{N}^{2}}{2}\right) \frac{\partial u_{N}}{\partial t} d x=-\varepsilon_{N} \int_{a}^{b} \frac{\partial}{\partial x}\left(Q_{N} u_{N}\right) \frac{\partial^{2} u_{N}}{\partial x \partial t} d x \\
= & -\varepsilon_{N} \int_{a}^{b} \frac{\partial}{\partial x}\left(Q_{N} u_{N}\right) \frac{\partial^{2}}{\partial x \partial t}\left(Q_{N} u_{N}\right) d x \\
& -\varepsilon_{N} \int_{a}^{b} \frac{\partial}{\partial x}\left(Q_{N} u_{N}\right) \frac{\partial^{2}}{\partial x \partial t}\left[\left(I-Q_{N}\right) u_{N}\right] d x \\
= & -\frac{\varepsilon_{N}}{2} \int_{a}^{b} \frac{\partial}{\partial t}\left\{\left[\frac{\partial}{\partial x}\left(Q_{N} u_{N}\right)\right]^{2}\right\} d x-\varepsilon_{N} \int_{a}^{b} \frac{\partial}{\partial x}\left(Q_{N} u_{N}\right) \frac{\partial^{2}}{\partial x \partial t}\left[\left(I-Q_{N}\right) u_{N}\right] d x \\
\leq & -\frac{\varepsilon_{N}}{2} \int_{a}^{b} \frac{\partial}{\partial t}\left\{\left[\frac{\partial}{\partial x}\left(Q_{N} u_{N}\right)\right]^{2}\right\} d x \\
& +\varepsilon_{N}\left\|\frac{\partial}{\partial x}\left(Q_{N} u_{N}\right)\right\| L_{L^{2}(a, b)}\left\|\frac{\partial^{2}}{\partial x \partial t}\left[\left(I-Q_{N}\right) u_{N}\right]\right\|_{L^{2}(a, b)}\left\|\frac{\partial u_{N}}{\partial t}\right\|_{L^{2}(a, b)} \\
\leq & -\frac{\varepsilon_{N}}{2} \int_{a}^{b} \frac{\partial}{\partial t}\left\{\left[\frac{\partial}{\partial x}\left(Q_{N} u_{N}\right)\right]^{2}\right\} d x+C \sqrt{\varepsilon_{N}}\left\|\frac{\partial}{\partial x}\left(Q_{N} u_{N}\right)\right\|_{L^{2}(a, b)} \\
\leq & -\frac{\varepsilon_{N}}{2} \int_{a}^{b} \frac{\partial}{\partial t}\left\{\left[\frac{\partial}{\partial x}\left(Q_{N} u_{N}\right)\right]^{2}\right\} d x+C\left\|\frac{\partial u_{N}}{\partial t}\right\|_{L^{2}(a, b)} \\
\leq & -\frac{\varepsilon_{N}}{2} \int_{a}^{b} \frac{\partial}{\partial t}\left\{\left[\frac{\partial}{\partial x}\left(Q_{N} u_{N}\right)\right]^{2}\right\} d x+C^{2}+\frac{1}{4}\left\|\frac{\partial u_{N}}{\partial t}\right\|_{L^{2}(a, b)}^{2} .
\end{aligned}
$$

Rearranging terms in the last expression, we obtain

$$
\begin{gathered}
\frac{3}{4}\left\|\frac{\partial u_{N}}{\partial t}\right\|_{L^{2}(a, b)}^{2}+\frac{\varepsilon_{N}}{2} \frac{d}{d t}\left\|\frac{\partial}{\partial x}\left(Q_{N} u_{N}\right)\right\|_{L^{2}(a, b)}^{2}-C \leq-\int_{a}^{b} \frac{\partial}{\partial x}\left(\frac{u_{N}^{2}}{2}\right) \frac{\partial u_{N}}{\partial t} d x \\
\leq\left\|\frac{\partial}{\partial x}\left(\frac{u_{N}^{2}}{2}\right)\right\|_{L^{2}(a, b)}\left\|\frac{\partial u_{N}}{\partial t}\right\|_{L^{2}(a, b)} \leq\left\|\frac{\partial}{\partial x}\left(\frac{u_{N}^{2}}{2}\right)\right\|_{L^{2}(a, b)}^{2}+\frac{1}{4}\left\|\frac{\partial u_{N}}{\partial t}\right\|_{L^{2}(a, b)}^{2}
\end{gathered}
$$

Rearranging terms again, we obtain

$$
\left\|\frac{\partial u_{N}}{\partial t}\right\|_{L^{2}(a, b)}^{2} \leq C-\varepsilon_{N} \frac{d}{d t}\left\|\frac{\partial}{\partial x}\left(Q_{N} u_{N}\right)\right\|_{L^{2}(a, b)}^{2}+2\left\|\frac{\partial}{\partial x}\left(\frac{u_{N}^{2}}{2}\right)\right\|_{L^{2}(a, b)}^{2} .
$$


Integrating over time, we obtain

$$
\begin{aligned}
\left\|\frac{\partial u_{N}}{\partial t}\right\|_{L^{2}\left(\Omega_{T}\right)}^{2} \leq & C+2 \int_{\Omega_{T}}\left|\frac{\partial}{\partial x}\left(\frac{u_{N}^{2}}{2}\right)\right|^{2} d x d t \\
& -\varepsilon_{N}\left\|\frac{\partial}{\partial x}\left(Q_{N} u_{N}(\cdot, T)\right)\right\|_{L^{2}(a, b)}^{2}+\varepsilon_{N}\left\|\frac{d}{d x}\left(Q_{N} g_{N}\right)\right\|_{L^{2}(a, b)}^{2} \\
\leq & C+2 \int_{U_{T}}\left|\frac{\partial}{\partial x}\left(\frac{u_{N}^{2}}{2}\right)\right|^{2} d x d t+\varepsilon_{N}\left\|\frac{d}{d x}\left(Q_{N} g_{N}\right)\right\|_{L^{2}(a, b)}^{2} \\
\leq & C+2 \int_{U_{T}}\left|\frac{\partial}{\partial x}\left(\frac{u_{N}^{2}}{2}\right)\right|^{2} d x d t+C \varepsilon_{N}\left\|\frac{d g_{N}}{d x}\right\|_{L^{2}(a, b)}^{2} \\
\leq & C+2 \int_{U_{T}}\left|\frac{\partial}{\partial x}\left(\frac{u_{N}^{2}}{2}\right)\right|^{2} d x d t+C \varepsilon_{N}\left\|\frac{d g}{d x}\right\|_{L^{2}(a, b)}^{2} .
\end{aligned}
$$

Now,

$$
\begin{aligned}
\int_{\Omega_{T}}\left|\frac{\partial}{\partial x}\left(\frac{u_{N}^{2}}{2}\right)\right|^{2} d x d t & =\int_{\Omega_{T}}\left|u_{N}\right|^{2}\left|\frac{\partial u_{N}}{\partial x}\right|^{2} d x d t \\
& \leq\left\|u_{N}\right\|_{L^{\infty}\left(\Omega_{T}\right)}^{2}\left\|\frac{\partial u_{N}}{\partial x}\right\|_{L^{2}\left(\Omega_{T}\right)}^{2} \leq \frac{C}{\varepsilon_{N}}
\end{aligned}
$$

Combining the last two results, we obtain $\varepsilon_{N}\left\|\frac{\partial u_{N}}{\partial t}\right\|_{L^{2}(a, b)}^{2} \leq C\left(1+\varepsilon_{N}+\varepsilon_{N}^{2}\right)$ so that

$$
\sqrt{\varepsilon_{N}}\left\|\frac{\partial u_{N}}{\partial t}\right\|_{L^{2}\left(\Omega_{T}\right)} \leq C
$$

Combining (3.22) and (3.23), we obtain

$$
\begin{array}{r}
\sqrt{\varepsilon_{N}}\left\|\operatorname{div} \mathbf{v}_{N}\right\|_{L^{2}\left(\Omega_{T}\right)}=\sqrt{\varepsilon_{N}}\left\|\frac{\partial u_{N}}{\partial t}+\frac{\partial}{\partial x}\left(\frac{u_{N}^{2}}{2}\right)\right\|_{L^{2}\left(\Omega_{T}\right)} \\
\leq \sqrt{\varepsilon_{N}}\left\|\frac{\partial u_{N}}{\partial t}\right\|_{L^{2}\left(\Omega_{T}\right)}+\sqrt{\varepsilon_{N}}\left\|\frac{\partial}{\partial x}\left(\frac{u_{N}^{2}}{2}\right)\right\|_{L^{2}\left(\Omega_{T}\right)} \leq C .
\end{array}
$$

3.5.2. $\left\{\operatorname{div} \mathbf{v}_{N}\right\}$ lies in a compact subset of $\boldsymbol{H}^{-1}\left(\boldsymbol{\Omega}_{\boldsymbol{T}}\right)$. Let $\widetilde{\varphi} \in H_{0}^{1}\left(\Omega_{T}\right)$. For all $t \in(0, T)$, let $\widetilde{\varphi}_{N}(\cdot, t) \in S_{p}^{N} \cap H_{0}^{1}(a, b)$ be the $H^{1}(a, b)$ projection of $\widetilde{\varphi}$ so that

$$
\int_{a}^{b} \frac{\partial \widetilde{\varphi}_{N}(\cdot, t)}{\partial x} \frac{\partial v}{\partial x} d x=\int_{a}^{b} \frac{\partial \widetilde{\varphi}(\cdot, t)}{\partial x} \frac{\partial v}{\partial x} d x \quad \forall v \in S_{p}^{N} \cap H_{0}^{1}(a, b) .
$$

We need the $H^{1}(a, b)$ projection into $S_{p}^{N}$ of an arbitrary $\widetilde{\varphi} \in H_{0}^{1}\left(\Omega_{T}\right)$ in order to use our FE formulation:

$$
\begin{aligned}
& \int_{\Omega_{T}}\left(\operatorname{div} \mathbf{v}_{N}\right) \widetilde{\varphi} d x d t=\int_{\Omega_{T}}\left(\operatorname{div} \mathbf{v}_{N}\right) \widetilde{\varphi}_{N} d x d t+\int_{\Omega_{T}}\left(\operatorname{div} \mathbf{v}_{N}\right)\left(\widetilde{\varphi}-\widetilde{\varphi}_{N}\right) d x d t \\
& =-\varepsilon_{N} \int_{\Omega_{T}} \frac{\partial}{\partial x}\left(Q_{N} u_{N}\right) \frac{\partial \widetilde{\varphi}_{N}}{\partial x} d x d t+\int_{\Omega_{T}}\left(\operatorname{div} \mathbf{v}_{N}\right)\left(\widetilde{\varphi}-\widetilde{\varphi}_{N}\right) d x d t \\
& =-\varepsilon_{N} \int_{\Omega_{T}} \frac{\partial}{\partial x}\left(Q_{N} u_{N}\right) \frac{\partial \widetilde{\varphi}}{\partial x} d x d t+\int_{\Omega_{T}}\left(\operatorname{div} \mathbf{v}_{N}\right)\left(\widetilde{\varphi}-\widetilde{\varphi}_{N}\right) d x d t
\end{aligned}
$$




$$
\begin{aligned}
& \leq \varepsilon_{N}\left\|\frac{\partial}{\partial x}\left(Q_{N} u_{N}\right)\right\|_{L^{2}\left(\Omega_{T}\right)}\left\|\frac{\partial \widetilde{\varphi}}{\partial x}\right\|_{L^{2}\left(\Omega_{T}\right)}+\left\|\operatorname{div} v_{N}\right\|_{L^{2}\left(\Omega_{T}\right)}\left\|\widetilde{\varphi}-\widetilde{\varphi}_{N}\right\|_{L^{2}\left(\Omega_{T}\right)} \\
& \leq \varepsilon_{N}\left\|\frac{\partial}{\partial x}\left(Q_{N} u_{N}\right)\right\|_{L^{2}\left(\Omega_{T}\right)}\left\|\frac{\partial \widetilde{\varphi}}{\partial x}\right\|_{L^{2}\left(\Omega_{T}\right)}+C h_{N}\left\|\operatorname{div} v_{N}\right\|_{L^{2}\left(\Omega_{T}\right)}\left\|\frac{\partial \widetilde{\varphi}}{\partial x}\right\|_{L^{2}\left(\Omega_{T}\right)} \\
& \leq C\left(\sqrt{\varepsilon_{N}}+\frac{h_{N}}{\sqrt{\varepsilon_{N}}}\right)\left\|\frac{\partial \widetilde{\varphi}}{\partial x}\right\|_{L^{2}\left(\Omega_{T}\right)}=C \sqrt{\varepsilon_{N}}\left(1+\frac{h_{N}}{\varepsilon_{N}}\right)\left\|\frac{\partial \widetilde{\varphi}}{\partial x}\right\|_{L^{2}\left(\Omega_{T}\right)} .
\end{aligned}
$$

From (3.8), we have that $\frac{h_{N}}{\varepsilon_{N}} \leq C$ so that

$$
\int_{\Omega_{T}}\left(\operatorname{div} \mathbf{v}_{N}\right) \widetilde{\varphi} d x d t \leq C \sqrt{\varepsilon_{N}}\left\|\frac{\partial \widetilde{\varphi}}{\partial x}\right\|_{L^{2}\left(\Omega_{T}\right)} .
$$

Let $\widetilde{\varphi} \in H_{0}^{1}\left(\Omega_{T}\right)$ with $\|\widetilde{\varphi}\|_{H^{1}\left(\Omega_{T}\right)} \leq 1$. Then, from (3.24), we have that

$$
\left\|\operatorname{div} \mathbf{v}_{N}\right\|_{H^{-1}\left(\Omega_{T}\right)} \leq C \sqrt{\varepsilon_{N}} \rightarrow 0 \text { as } N \rightarrow \infty
$$

so that $\left\{\operatorname{div} \mathbf{v}_{N}\right\}$ lies in a compact subset of $H^{-1}\left(\Omega_{T}\right)$.

3.5.3. $\left\{\operatorname{curl}_{N}\right\}$ lies in a compact subset of $\boldsymbol{H}^{-1}\left(\Omega_{T}\right)$. Let $\varphi \in C_{0}^{\infty}\left(\Omega_{T}\right)$ be a test function. Since $u_{N} \varphi \in H_{0}^{1}\left(\Omega_{T}\right)$, we can choose $\widetilde{\varphi}=u_{N} \varphi$ in (3.24). Then,

$$
\begin{aligned}
& \int_{\Omega_{T}}\left(\operatorname{curl} \mathbf{w}_{N}\right) \varphi d x d t=\int_{\Omega_{T}}\left(\operatorname{div} \mathbf{v}_{N}\right) u_{N} \varphi d x d t \\
& \leq C \sqrt{\varepsilon_{N}}\left\|\frac{\partial}{\partial x}\left(u_{N} \varphi\right)\right\|_{L^{2}\left(\Omega_{T}\right)}=C \sqrt{\varepsilon_{N}}\left\|u_{N} \frac{\partial \varphi}{\partial x}+\varphi \frac{\partial u_{N}}{\partial x}\right\|_{L^{2}\left(\Omega_{T}\right)} \\
& \leq C \sqrt{\varepsilon_{N}}\left(\left\|u_{N} \frac{\partial \varphi}{\partial x}\right\|_{L^{2}\left(\Omega_{T}\right)}+\left\|\varphi \frac{\partial u_{N}}{\partial x}\right\|_{L^{2}\left(\Omega_{T}\right)}\right) \\
& \leq C \sqrt{\varepsilon_{N}}\left(\left\|u_{N}\right\|_{L^{\infty}\left(\Omega_{T}\right)}\left\|\frac{\partial \varphi}{\partial x}\right\|_{L^{2}\left(\Omega_{T}\right)}+\|\varphi\|_{L^{\infty}\left(\Omega_{T}\right)}\left\|\frac{\partial u_{N}}{\partial x}\right\|_{L^{2}\left(\Omega_{T}\right)}\right) \\
& \leq C\left(\sqrt{\varepsilon_{N}}\left\|\frac{\partial \varphi}{\partial x}\right\|_{L^{2}\left(\Omega_{T}\right)}+\|\varphi\|_{L^{\infty}\left(\Omega_{T}\right)}\right) .
\end{aligned}
$$

Using a variational form of Murat's lemma $[13,19]$ gives, from the last result, that $\left\{\operatorname{curl} \mathbf{w}_{N}\right\}$ lies in a compact subset of $H^{-1}\left(\Omega_{T}\right)$.

3.5.4. Strong convergence in $L^{2}\left(\Omega_{T}\right)$ of a subsequence of $\left\{u_{N}\right\}$. Since $\left\|u_{N}\right\|_{L^{\infty}\left(\Omega_{T}\right)} \leq C$, there exists a subsequence $\left\{u_{N_{k}}\right\}$ of $\left\{u_{N}\right\}$ such that for $1 \leq p \leq 4$, $\left\{u_{N_{k}}^{p}\right\}$ converges weakly in $L^{2}\left(\Omega_{T}\right)$. Let $\overline{u^{(p)}} \in L^{2}\left(\Omega_{T}\right)$ be the weak limit of $u_{N_{k}}^{p}$. Then, $\mathbf{v}_{N_{k}}$ and $\mathbf{w}_{N_{k}}$ converge weakly:

$$
\mathbf{v}_{N_{k}} \rightarrow\left(\frac{\overline{u^{(2)}}}{2}, \overline{u^{(1)}}\right)=: \overline{\mathbf{v}} \quad \text { and } \quad \mathbf{w}_{N_{k}}-\left(\frac{\overline{u^{(2)}}}{2},-\frac{\overline{u^{(3)}}}{3}\right)=: \overline{\mathbf{w}} .
$$

By the div-curl lemma $[7,21,28]$, we have

$$
\lim _{k \rightarrow \infty} \int_{\Omega_{T}}\left(\mathbf{v}_{N_{k}} \cdot \mathbf{w}_{N_{k}}\right) \varphi d x d t=\int_{\Omega_{T}}(\overline{\mathbf{v}} \cdot \overline{\mathbf{w}}) \varphi d x d t \quad \forall \varphi \in C_{0}^{\infty}\left(\Omega_{T}\right) .
$$

For all $\varphi \in C_{0}^{\infty}\left(\Omega_{T}\right)$,

$$
\begin{gathered}
\lim _{k \rightarrow \infty} \int_{\Omega_{T}}\left(\mathbf{v}_{N_{k}} \cdot \mathbf{w}_{N_{k}}\right) \varphi d x d t=\lim _{k \rightarrow \infty} \int_{\Omega_{T}}\left(\frac{u_{N_{k}}{ }^{4}}{4}-\frac{u_{N_{k}}{ }^{4}}{3}\right) \varphi d x d t \\
=\lim _{k \rightarrow \infty} \int_{\Omega_{T}}-\frac{u_{N_{k}}{ }^{4}}{12} \varphi d x d t=\int_{\Omega_{T}}-\frac{u^{(4)}}{12} \varphi d x d t .
\end{gathered}
$$


For the right-hand side of (3.25) we have

$$
\int_{\Omega_{T}}(\overline{\mathbf{v}} \cdot \overline{\mathbf{w}}) \varphi d x d t=\int_{\Omega_{T}}\left(\frac{1}{4}\left(\overline{u^{(2)}}\right)^{2}-\frac{1}{3} \overline{u^{(1)}} \overline{u^{(3)}}\right) \varphi d x d t .
$$

Combining (3.25)-(3.27) yields that

$$
\overline{u^{(4)}}=4 \overline{u^{(1)}} \overline{u^{(3)}}-3\left(\overline{u^{(2)}}\right)^{2} \text { a.e., }
$$

which can be used to show that $u_{N_{k}}$ converges strongly to $\overline{u^{(1)}}$ in $L^{2}\left(\Omega_{T}\right)$. First, we have

$$
\left(u_{N_{k}}-\overline{u^{(1)}}\right)^{4}=u_{N_{k}}{ }^{4}-4 u_{N_{k}}{ }^{3} \overline{u^{(1)}}+6{u_{N_{k}}}^{2}\left(\overline{u^{(1)}}\right)^{2}-4 u_{N_{k}}\left(\overline{u^{(1)}}\right)^{3}+\left(\overline{u^{(1)}}\right)^{4} .
$$

Taking the weak limit of both sides of the last equation, we have that

$$
\begin{aligned}
w & -\lim _{k \rightarrow \infty}\left(u_{N_{k}}-\overline{u^{(1)}}\right)^{4} \\
& =\overline{u^{(4)}}-4 \overline{u^{(3)}} \overline{u^{(1)}}+6 \overline{u^{(2)}}\left(\overline{u^{(1)}}\right)^{2}-4\left(\overline{u^{(1)}}\right)^{4}+\left(\overline{u^{(1)}}\right)^{4} \\
& =4 \overline{u^{(1)}} \overline{u^{(3)}}-3\left(\overline{u^{(2)}}\right)^{2}-4 \overline{u^{(3)}} \overline{u^{(1)}}+6 \overline{u^{(2)}}\left(\overline{u^{(1)}}\right)^{2}-4\left(\overline{u^{(1)}}\right)^{4}+\left(\overline{u^{(1)}}\right)^{4} \\
& =-3\left(\overline{u^{(2)}}\right)^{2}+6 \overline{u^{(2)}}\left(\overline{u^{(1)}}\right)^{2}-3\left(\overline{u^{(1)}}\right)^{4}=-3\left[\overline{u^{(2)}}-\left(\overline{u^{(1)}}\right)^{2}\right]^{2} \leq 0 .
\end{aligned}
$$

Then

$$
0 \leq \lim _{k \rightarrow \infty} \int_{\Omega_{T}}\left(u_{N_{k}}-\overline{u^{(1)}}\right)^{4} d x d t=\int_{\Omega_{T}}-3\left[\overline{u^{(2)}}-\left(\overline{u^{(1)}}\right)^{2}\right]^{2} d x d t \leq 0 .
$$

We now have $\overline{u^{(2)}}=\left(\overline{u^{(1)}}\right)^{2}$ a.e., which gives us

$$
\left\|\overline{u^{(1)}}\right\|_{L^{2}\left(\Omega_{T}\right)}^{2}=\int_{\Omega_{T}} \overline{u^{(2)}} d x d t=\lim _{k \rightarrow \infty} \int_{\Omega_{T}} u_{N_{k}}{ }^{2} d x d t=\lim _{k \rightarrow \infty}\left\|u_{N_{k}}\right\|_{L^{2}\left(\Omega_{T}\right)}^{2} .
$$

Therefore, $\bar{u}:=\overline{u^{(1)}}$ is the strong limit of $u_{N_{k}}$ in $L^{2}\left(\Omega_{T}\right)$.

3.6. Convergence to a solution of the hyperbolic conservation law. We now show that $\bar{u}$ is a solution of the conservation law (3.4). For all test functions $\varphi \in C_{0}^{\infty}\left(\Omega_{T}\right)$,

$$
\begin{aligned}
& \int_{\Omega_{T}} {\left[\frac{\partial \bar{u}}{\partial t} \varphi+\frac{\partial}{\partial x}\left(\frac{\bar{u}^{2}}{2}\right) \varphi\right] d x d t=-\int_{\Omega_{T}}\left[\bar{u} \frac{\partial \varphi}{\partial t}+\frac{\bar{u}^{2}}{2} \frac{\partial \varphi}{\partial x}\right] d x d t } \\
&=-\int_{\Omega_{T}}\left[\overline{u^{(1)}} \frac{\partial \varphi}{\partial t}+\frac{\overline{u^{(2)}}}{2} \frac{\partial \varphi}{\partial x}\right] d x d t=-\lim _{k \rightarrow \infty} \int_{\Omega_{T}}\left[u_{N_{k}} \frac{\partial \varphi}{\partial t}+\frac{u_{N_{k}}^{2}}{2} \frac{\partial \varphi}{\partial x}\right] d x d t \\
&=\lim _{k \rightarrow \infty} \int_{\Omega_{T}}\left[\frac{\partial u_{N_{k}}}{\partial t} \varphi+\frac{\partial}{\partial x}\left(\frac{u_{N_{k}}^{2}}{2}\right) \varphi\right] d x d t=\lim _{k \rightarrow \infty} \int_{\Omega_{T}}\left(\operatorname{div} \mathbf{v}_{N_{k}}\right) \varphi d x d t .
\end{aligned}
$$

The right-hand side of last expression vanishes since

$$
\begin{gathered}
0 \leq\left|\int_{\Omega_{T}}\left(\operatorname{div} \mathbf{v}_{N_{k}}\right) \varphi d x d t\right| \leq\left\|\operatorname{div} \mathbf{v}_{N_{k}}\right\|_{H^{-1}\left(\Omega_{T}\right)}\|\varphi\|_{H^{1}\left(\Omega_{T}\right)} \\
\leq C \sqrt{\varepsilon_{N}}\|\varphi\|_{H^{1}\left(\Omega_{T}\right)} \rightarrow 0 \text { as } k \rightarrow \infty
\end{gathered}
$$

so that $\bar{u}$ is a solution of (3.4). 
3.7. Convergence to the entropy solution. We showed in sections 3.5 and 3.6 that $\left\{u_{N_{k}}\right\}$ converges strongly to a solution $\bar{u}$ of the conservation law. We now show that, if the strengthened requirements (3.11) and (3.12) are satisfied, then $\bar{u}$ is the physically relevant entropy solution.

Let $\varphi \in C_{0}^{\infty}\left(\Omega_{T}\right)$; then

$$
\begin{aligned}
& \left|\int_{\Omega_{T}}\left(\bar{u}^{3}-u_{N_{k}}{ }^{3}\right) \varphi d x d t\right|=\left|\int_{\Omega_{T}}\left(\bar{u}-u_{N_{k}}\right)\left(\bar{u}^{2}+\bar{u} u_{N_{k}}+u_{N_{k}}{ }^{2}\right) \varphi d x d t\right| \\
& \quad \leq\left\|\bar{u}-u_{N_{k}}\right\|_{L^{2}\left(\Omega_{T}\right)}\left\|\left(\bar{u}^{2}+\bar{u} u_{N_{k}}+u_{N_{k}}{ }^{2}\right) \varphi\right\|_{L^{2}\left(\Omega_{T}\right)} \\
& \quad \leq\|\varphi\|_{L^{\infty}\left(\Omega_{T}\right)}\left\|\bar{u}-u_{N_{k}}\right\|_{L^{2}\left(\Omega_{T}\right)}\left\|\bar{u}^{2}+\bar{u} u_{N_{k}}+u_{N_{k}}{ }^{2}\right\|_{L^{2}\left(\Omega_{T}\right)} \\
& \quad \leq\|\varphi\|_{L^{\infty}\left(\Omega_{T}\right)}\left\|\bar{u}-u_{N_{k}}\right\|_{L^{2}\left(\Omega_{T}\right)}\left(\left\|\bar{u}^{2}\right\|_{L^{2}\left(\Omega_{T}\right)}+\left\|\bar{u} u_{N_{k}}\right\|_{L^{2}\left(\Omega_{T}\right)}+\left\|u_{N_{k}}{ }^{2}\right\|_{L^{2}\left(\Omega_{T}\right)}\right) \\
& \quad \leq\|\varphi\|_{L^{\infty}\left(\Omega_{T}\right)}\left\|\bar{u}-u_{N_{k}}\right\|_{L^{2}\left(\Omega_{T}\right)} \\
& \quad\left(\|\bar{u}\|_{L^{4}\left(\Omega_{T}\right)}^{2}+\left\|u_{N_{k}}\right\|_{L^{\infty}\left(\Omega_{T}\right)}\|\bar{u}\|_{L^{2}\left(\Omega_{T}\right)}+\left\|u_{N_{k}}\right\|_{L^{\infty}\left(\Omega_{T}\right)}^{2} \sqrt{\left|\Omega_{T}\right|}\right) .
\end{aligned}
$$

Since $\left\{u_{N}\right\}$ is uniformly bounded, we have that $\left\|u_{N_{k}}\right\|_{L^{\infty}\left(\Omega_{T}\right)} \leq C$. Also, $\bar{u}$ is in $L^{4}\left(\Omega_{T}\right)$ since $\bar{u}^{2}=\overline{u^{(2)}} \in L^{2}\left(\Omega_{T}\right)$. Then, since $\lim _{k \rightarrow \infty}\left\|\bar{u}-u_{N_{k}}\right\|_{L^{2}\left(\Omega_{T}\right)}=0$, we have that

$$
\lim _{k \rightarrow \infty} \int_{\Omega_{T}}\left(u_{N_{k}}\right)^{3} \varphi d x d t=\int_{\Omega_{T}} \bar{u}^{3} \varphi d x d t
$$

Now, let $\varphi \in C_{0}^{\infty}\left(\Omega_{T}\right)$ with $\varphi \geq 0$; then,

$$
\begin{aligned}
\int_{\Omega_{T}}\left[\frac{\partial}{\partial t}\right. & \left.\left(\frac{\bar{u}^{2}}{2}\right)+\frac{\partial}{\partial x}\left(\frac{\bar{u}^{3}}{3}\right)\right] \varphi d x d t=-\int_{\Omega_{T}}\left(\frac{\bar{u}^{2}}{2} \frac{\partial \varphi}{\partial t}+\frac{\bar{u}^{3}}{3} \frac{\partial \varphi}{\partial x}\right) d x d t \\
& =-\lim _{k \rightarrow \infty} \int_{\Omega_{T}}\left(\frac{u_{N_{k}}{ }^{2}}{2} \frac{\partial \varphi}{\partial t}+\frac{u_{N_{k}}{ }^{3}}{3} \frac{\partial \varphi}{\partial x}\right) d x d t \\
& =\lim _{k \rightarrow \infty} \int_{\Omega_{T}}\left[\frac{\partial}{\partial t}\left(\frac{u_{N_{k}}{ }^{2}}{2}\right)+\frac{\partial}{\partial x}\left(\frac{u_{N_{k}}{ }^{3}}{3}\right)\right] \varphi d x d t \\
& =\lim _{k \rightarrow \infty} \int_{\Omega_{T}}\left(\operatorname{curl} \mathbf{w}_{N_{k}}\right) \varphi d x d t=\lim _{k \rightarrow \infty} \int_{\Omega_{T}}\left(\operatorname{div} \mathbf{v}_{N_{k}}\right) u_{N_{k}} \varphi d x d t
\end{aligned}
$$

Let $z_{N}=u_{N} \varphi$. For all $t \in(0, T)$, let $z_{N}^{h}(\cdot, t) \in S_{p}^{N} \cap H_{0}^{1}(a, b)$ be the $H^{1}(a, b)$ projection of $z_{N}$ so that, for all $v \in S_{p}^{N} \cap H_{0}^{1}(a, b)$,

$$
\int_{a}^{b} \frac{\partial z_{N}^{h}(\cdot, t)}{\partial x} \frac{\partial v}{\partial x} d x=\int_{a}^{b} \frac{\partial z_{N}(\cdot, t)}{\partial x} \frac{\partial v}{\partial x} d x
$$


We then show that, as $k \rightarrow \infty$, the right-hand side of (3.28) is nonpositive:

$$
\begin{aligned}
& \int_{\Omega_{T}}\left(\operatorname{div} u_{N}\right) u_{N} \varphi d x d t=\int_{\Omega_{T}}\left(\operatorname{div} u_{N}\right) z_{N} d x d t \\
& =\int_{\Omega_{T}}\left(\operatorname{div} u_{N}\right) z_{N}^{h} d x d t+\int_{\Omega_{T}}\left(\operatorname{div} u_{N_{k}}\right)\left(z_{N}-z_{N}^{h}\right) d x d t \\
& =-\varepsilon_{N} \int_{\Omega_{T}} \frac{\partial z_{N}^{h}}{\partial x} \frac{\partial}{\partial x}\left(Q_{N} u_{N}\right) d x d t+\int_{\Omega_{T}}\left(\operatorname{div} u_{N}\right)\left(z_{N}-z_{N}^{h}\right) d x d t \\
& =-\varepsilon_{N} \int_{\Omega_{T}} \frac{\partial z_{N}}{\partial x} \frac{\partial}{\partial x}\left(Q_{N} u_{N}\right) d x d t+\int_{\Omega_{T}}\left(\operatorname{div} u_{N}\right)\left(z_{N}-z_{N}^{h}\right) d x d t \\
& =-\varepsilon_{N} \int_{\Omega_{T}} \frac{\partial z_{N}}{\partial x} \frac{\partial u_{N}}{\partial x} d x d t+\varepsilon_{N} \int_{\Omega_{T}} \frac{\partial z_{N}}{\partial x} \frac{\partial}{\partial x}\left[\left(I-Q_{N}\right) u_{N}\right] d x d t \\
& +\int_{\Omega_{T}}\left(\operatorname{div} u_{N}\right)\left(z_{N}-z_{N}^{h}\right) d x d t \\
& =-\varepsilon_{N} \int_{\Omega_{T}} \frac{\partial}{\partial x}\left(u_{N} \varphi\right) \frac{\partial u_{N}}{\partial x} d x d t \\
& +\varepsilon_{N} \int_{\Omega_{T}} \frac{\partial z_{N}}{\partial x} \frac{\partial}{\partial x}\left[\left(I-Q_{N}\right) u_{N}\right] d x d t+\int_{\Omega_{T}}\left(\operatorname{div} u_{N}\right)\left(z_{N}-z_{N}^{h}\right) d x d t \\
& =-\varepsilon_{N} \int_{\Omega_{T}} \varphi\left|\frac{\partial u_{N}}{\partial x}\right|^{2} d x d t-\varepsilon_{N} \int_{\Omega_{T}} u_{N} \frac{\partial \varphi}{\partial x} \frac{\partial u_{N}}{\partial x} d x d t \\
& +\varepsilon_{N} \int_{\Omega_{T}} \frac{\partial z_{N}}{\partial x} \frac{\partial}{\partial x}\left[\left(I-Q_{N}\right) u_{N}\right] d x d t+\int_{\Omega_{T}}\left(\operatorname{div} u_{N}\right)\left(z_{N}-z_{N}^{h}\right) d x d t .
\end{aligned}
$$

For the second term on the right-hand side of (3.29), we have that

$$
\begin{gathered}
\left|-\varepsilon_{N} \int_{\Omega_{T}} u_{N} \frac{\partial \varphi}{\partial x} \frac{\partial u_{N}}{\partial x} d x d t\right| \leq \varepsilon_{N}\left\|u_{N}\right\|_{L^{\infty}\left(\Omega_{T}\right)}\left\|\frac{\partial u_{N}}{\partial x}\right\|_{L^{2}\left(\Omega_{T}\right)}\left\|\frac{\partial \varphi}{\partial x}\right\|_{L^{2}\left(\Omega_{T}\right)} \\
\leq C \sqrt{\varepsilon_{N}}\left\|\frac{\partial \varphi}{\partial x}\right\|_{L^{2}\left(\Omega_{T}\right)} \rightarrow 0 \text { as } N \rightarrow \infty .
\end{gathered}
$$

For the third term on the right-hand side of (3.29), we have that

$$
\begin{aligned}
& \left|\varepsilon_{N} \int_{\Omega_{T}} \frac{\partial z_{N}}{\partial x} \frac{\partial}{\partial x}\left[\left(I-Q_{N}\right) u_{N}\right] d x d t\right| \leq \varepsilon_{N}\left\|\frac{\partial z_{N}}{\partial x}\right\|_{L^{2}\left(\Omega_{T}\right)}\left\|\frac{\partial}{\partial x}\left[\left(I-Q_{N}\right) u_{N}\right]\right\|_{L^{2}\left(\Omega_{T}\right)} \\
& =\varepsilon_{N}\left\|\frac{\partial}{\partial x}\left(u_{N} \varphi\right)\right\|_{L^{2}\left(\Omega_{T}\right)}\left\|\frac{\partial}{\partial x}\left[\left(I-Q_{N}\right) u_{N}\right]\right\|_{L^{2}\left(\Omega_{T}\right)} \leq \varepsilon_{N}\left\|\frac{\partial}{\partial x}\left[\left(I-Q_{N}\right) u_{N}\right]\right\|_{L^{2}\left(\Omega_{T}\right)} \\
& \quad\left(\|\varphi\|_{L^{\infty}\left(\Omega_{T}\right)}\left\|\frac{\partial u_{N}}{\partial x}\right\|_{L^{2}\left(\Omega_{T}\right)}+\left\|u_{N}\right\|_{L^{\infty}\left(\Omega_{T}\right)}\left\|\frac{\partial \varphi}{\partial x}\right\|_{L^{2}\left(\Omega_{T}\right)}\right) \\
& \leq C \sqrt{\varepsilon_{N}}\left\|\frac{\partial}{\partial x}\left[\left(I-Q_{N}\right) u_{N}\right]\right\|_{L^{2}\left(\Omega_{T}\right)}\left(\|\varphi\|_{L^{\infty}\left(\Omega_{T}\right)}+\sqrt{\varepsilon_{N}}\left\|\frac{\partial \varphi}{\partial x}\right\|_{L^{2}\left(\Omega_{T}\right)}\right) \rightarrow 0 \text { as } N \rightarrow \infty .
\end{aligned}
$$

For the fourth term on the right-hand side of (3.29), we have that

$$
\begin{aligned}
& \left|\int_{\Omega_{T}}\left(\operatorname{div} u_{N}\right)\left(z_{N}-z_{N}^{h}\right) d x d t\right| \leq\left\|\operatorname{div} u_{N}\right\|_{L^{2}\left(\Omega_{T}\right)}\left\|z_{N}-z_{N}^{h}\right\|_{L^{2}\left(\Omega_{T}\right)} \\
& \leq C h_{N}\left\|\operatorname{div} u_{N}\right\|_{L^{2}\left(\Omega_{T}\right)}\left\|\frac{\partial z_{N}}{\partial x}\right\|_{L^{2}\left(\Omega_{T}\right)} \leq C \frac{h_{N}}{\sqrt{\varepsilon_{N}}}\left\|\frac{\partial z_{N}}{\partial x}\right\|_{L^{2}\left(\Omega_{T}\right)}
\end{aligned}
$$




$$
\begin{aligned}
& =C \frac{h_{N}}{\sqrt{\varepsilon_{N}}}\left\|\frac{\partial}{\partial x}\left(u_{N} \varphi\right)\right\|_{L^{2}\left(\Omega_{T}\right)} \\
& \leq C \frac{h_{N}}{\sqrt{\varepsilon_{N}}}\left(\left\|u_{N}\right\|_{L^{\infty}\left(\Omega_{T}\right)}\left\|\frac{\partial \varphi}{\partial x}\right\|_{L^{2}\left(\Omega_{T}\right)}+\|\varphi\|_{L^{\infty}\left(\Omega_{T}\right)}\left\|\frac{\partial u_{N}}{\partial x}\right\|_{L^{2}\left(\Omega_{T}\right)}\right) \\
& \leq C\left(\frac{h_{N}}{\sqrt{\varepsilon_{N}}}\left\|\frac{\partial \varphi}{\partial x}\right\|_{L^{2}\left(\Omega_{T}\right)}+\frac{h_{N}}{\varepsilon_{N}}\|\varphi\|_{L^{\infty}\left(\Omega_{T}\right)}\right) \rightarrow 0 \text { as } N \rightarrow \infty .
\end{aligned}
$$

Thus, we have shown that the second, third, and fourth terms on the right-hand side of (3.29) vanish as $N \rightarrow \infty$. Since the first term is clearly nonpositive, we have that

$$
\int_{\Omega_{T}}\left[\frac{\partial}{\partial t}\left(\frac{\bar{u}^{2}}{2}\right)+\frac{\partial}{\partial x}\left(\frac{\bar{u}^{3}}{3}\right)\right] \varphi d x d t=\liminf _{k \rightarrow \infty} \int_{\Omega_{T}}\left(\operatorname{div} u_{N_{k}}\right) u_{N_{k}} \varphi d x d t \leq 0
$$

so that the $\bar{u}$ is the entropy solution of (3.4). This completes the proof of Theorem 3.1 .

3.8. Verifying the hypotheses of Theorem 3.1. In the hierarchical finite element formulation (3.6), we have to choose $\varepsilon_{N}, m$, and the form of $Q_{N}$ for $k \leq m$. Let $0<\delta \leq \theta \leq 1$. We then choose

$$
\varepsilon_{N}=C h_{N}{ }^{\theta}, \quad m_{H} \leq \frac{\delta N}{2}, \quad \text { and } \quad Q_{k, i}= \begin{cases}0, & k \leq m_{H}, \\ 1, & k>m_{H} .\end{cases}
$$

It is then evident that $\varepsilon_{N}, h_{N} \rightarrow 0$ as $N \rightarrow \infty$ so (3.7) holds. Since $0<\theta \leq 1$, $\frac{\varepsilon_{N}}{h_{N}}=C h_{N}{ }^{\theta-1} \geq C$ so that (3.8) also holds.

Let $v \in S_{p}^{N} ;\left(I-Q_{N}\right)$ is simply an interpolation operator on a coarse grid so that

$$
\left\|\left(I-Q_{N}\right) v\right\|_{L^{2}(a, b)} \leq C\|v\|_{L^{2}(a, b)} .
$$

$Q_{N}$ retains the high frequencies of a function, so $\left(I-Q_{N}\right)$ eliminates them: $Q_{k, i}=$ $1 \Rightarrow 1-Q_{k, i}=0$ for $k>m_{H}$ so that $\left(I-Q_{N}\right) v \in S_{p}^{m_{H}}$. Using a standard inverse estimate,

$$
\begin{aligned}
& \left\|\frac{\partial}{\partial x}\left[\left(I-Q_{N}\right) u_{N}\right]\right\|_{L^{2}(a, b)} \leq C\left(h_{m_{H}}\right)^{-1}\left\|\left(I-Q_{N}\right) v\right\|_{L^{2}(a, b)} \\
& \leq C M^{m_{H}}\left\|\left(I-Q_{N}\right) v\right\|_{L^{2}(a, b)} \leq C M^{\frac{N \delta}{2}}\left\|\left(I-Q_{N}\right) v\right\|_{L^{2}(a, b)} \\
& \leq C\left(\frac{\nu}{h_{N}}\right)^{\frac{\delta}{2}}\left\|\left(I-Q_{N}\right) v\right\|_{L^{2}(a, b)} \leq C\left(\frac{\nu}{h_{N}}\right)^{\frac{\delta}{2}}\|v\|_{L^{2}(a, b)} \\
& =C \sqrt{\nu^{\delta}} h_{N}{ }^{-\frac{\delta}{2}}\|v\|_{L^{2}(a, b)}
\end{aligned}
$$

and

$$
\begin{aligned}
\sqrt{\varepsilon_{N}}\left\|\frac{\partial}{\partial x}\left[\left(I-Q_{N}\right) u_{N}\right]\right\|_{L^{2}(a, b)} & \leq C \sqrt{\nu^{\delta}} \sqrt{\frac{\varepsilon_{N}}{h_{N}^{\delta}}}\|v\|_{L^{2}(a, b)} \\
& =C \sqrt{{h_{N}}^{\theta-\delta}}\|v\|_{L^{2}(a, b)} .
\end{aligned}
$$

Since $\delta \leq \theta$, we have that $h_{N}{ }^{\theta-\delta} \leq C$; therefore, (3.9) is satisfied.

Since $\left(I-Q_{N}\right)$ is an interpolation operator on a coarse grid, for all $v \in S_{p}^{N}$,

$$
\left\|\frac{d}{d x}\left(Q_{N} v\right)\right\|_{L^{2}(a, b)}=\left\|\frac{d v}{d x}-\frac{d}{d x}\left[\left(I-Q_{N}\right) v\right]\right\|_{L^{2}(a, b)} \leq C\left\|\frac{d v}{d x}\right\|_{L^{2}(a, b)} .
$$


Therefore, (3.10) is satisfied. This completes the verification of the hypotheses (3.7)(3.10) of Theorem 3.1 that are used to prove the convergence of the hierarchical finite element approximations.

To verify the hypotheses (3.11) and (3.12) of Theorem 3.1 that are used to prove the convergence of the hierarchical finite element approximations to the entropy solution, we must choose $0<\theta<1$. In this case, $\frac{\varepsilon_{N}}{h_{N}}=C h_{N}{ }^{\theta-1} \rightarrow \infty$ as $N \rightarrow \infty$ so that (3.11) holds. Since now $\delta<\theta$ so that $h_{N}{ }^{\theta-\delta} \rightarrow 0$ as $N \rightarrow \infty$, (3.30) implies that (3.12) holds.

4. A simple computational illustration. We consider the simple periodic problem for the Burgers equation in one dimension:

$$
\left\{\begin{array}{l}
\frac{\partial u}{\partial t}+\frac{\partial}{\partial x}\left(\frac{u^{2}}{2}\right)=0 \text { on }(-1,+1) \times(0, T) \\
u(-1, t)=u(+1, t) \text { for all } t \in(0, T) \\
u(x, 0)=1+\frac{1}{2} \sin (\pi x) .
\end{array}\right.
$$

A means for establishing the exact solution of this problem is given in $[8,16]$. All of our numerical results were generated using the finite element library deal. II [2]. More extensive computational experimentations are provided in $[3,4]$.

In (2.1), the values of several parameters must be chosen. We set $\varepsilon_{N}=h_{N}$ and add diffusion only to the finest level: $Q_{N ; n, \imath}=0$ for $n<N$. Neither of these choices satisfies the requirements of Theorem 3.1 for convergence to the entropy solution. Even with the smaller diffusion term, however, our numerical experiments indicate that the approximations still converge to the correct solution.

After spatial discretization is effected using linear hierarchical finite element functions, the resulting system of ordinary differential equations is integrated using a thirdorder, strong, stability-preserving Runge-Kutta method found in [11], with time step $\Delta t$ that satisfies the CFL condition $\Delta t / h_{N} \sup u \leq 0.2$.

The exact and discrete solutions of (4.1) are given in Figure 5. Because we are approximating a discontinuous solution with continuous piecewise polynomials, we see Gibbs oscillations near the discontinuity; see Figure 5. A simple post-processing strategy to remove the oscillations is to set the coefficients of the hierarchical expansion to zero around the discontinuity. The question then becomes how to determine the location of the discontinuity. Let $\beta_{n+1, \imath}$ be a high frequency hierarchical coefficient in the discrete solution. Let $\beta_{n, j}$ be the parent hierarchical coefficient, so the support of $\psi_{n+1, \imath}$ is a subset of $\psi_{n, \jmath}$. If the solution is continuously differentiable in the region of the support of $\psi_{n, \jmath}$, then $\beta_{n, j} / \beta_{n+1,2} \approx 2$. Thus, our simple post-processing strategy is as follows: For the highest four frequencies, if a hierarchical coefficient is larger than half the value of its parent, then it is set to zero. Our simple post-processing strategy only affects the region around a discontinuity, but it has the disadvantage of smoothing across the discontinuity. See Figure 6. We note that post-processing strategies must also be applied in the spectral viscosity method in order to reduce the size of the Gibbs oscillations.

In Table 1, we use the $L^{1}$ norm to measure errors in the approximate solution. Near a discontinuity, we are limited to how well a piecewise polynomial can approximate a solution. We are more interested in the convergence rates in the smooth regions. We therefore exclude a region of length 0.2 around the discontinuity in our error calculations. We see that away from the discontinuity, we achieve the optimal 

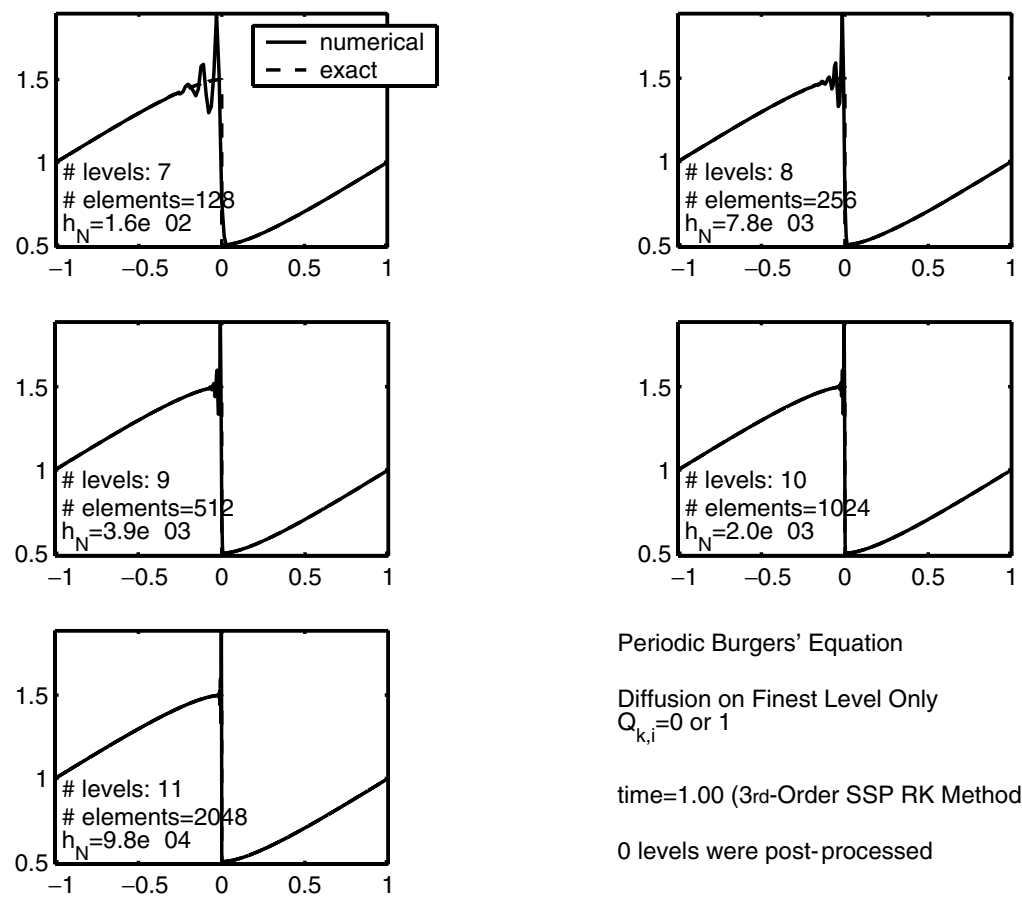

Periodic Burgers' Equation

Diffusion on Finest Level Only $\mathrm{Q}_{\mathrm{k}, \mathrm{i}}=0$ or 1

time $=1.00$ (3rd-Order SSP RK Method)

0 levels were post-processed

FIG. 5. Solution of periodic Burgers equation with linear polynomials (without post-processing).
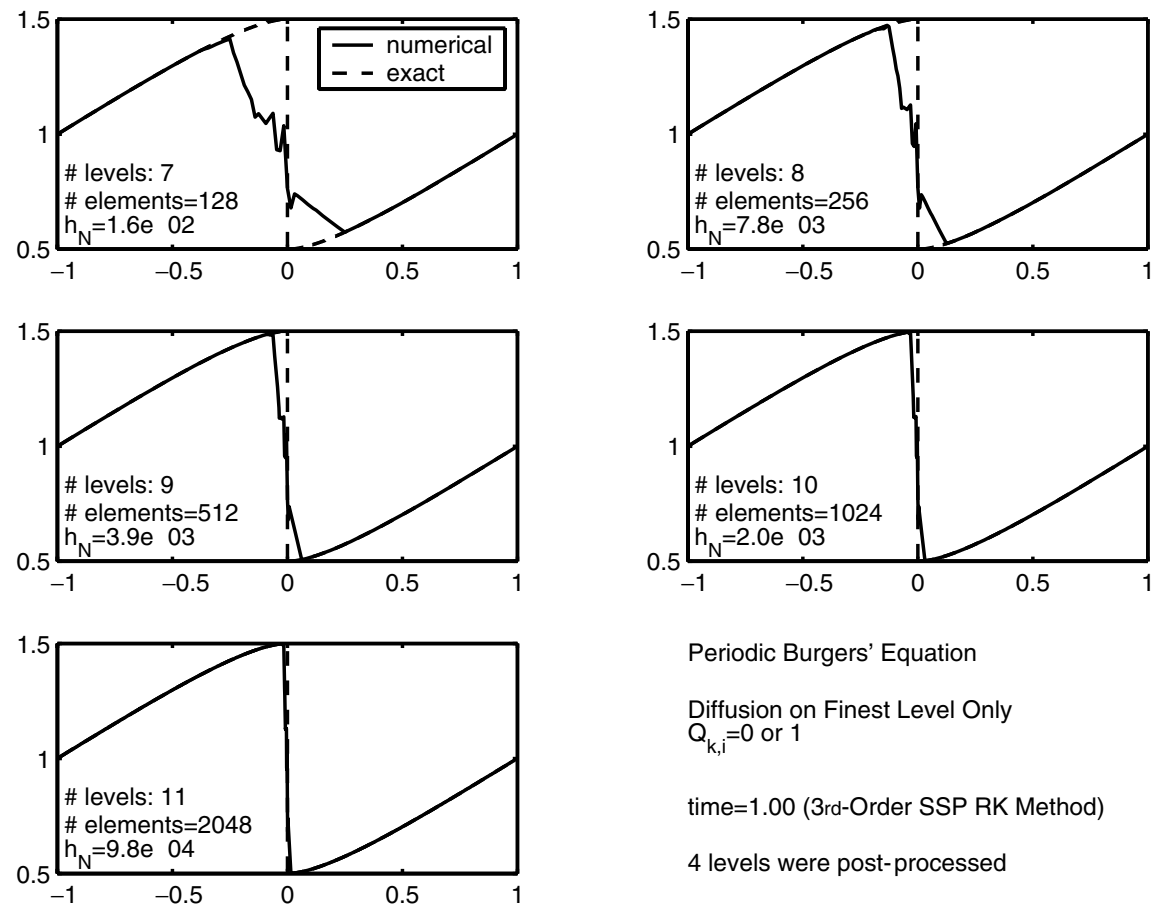

Periodic Burgers' Equation

Diffusion on Finest Level Only $\mathrm{Q}_{\mathrm{k}, \mathrm{i}}=0$ or 1

time $=1.00(3 r d-O r d e r$ SSP RK Method $)$

4 levels were post-processed

FIG. 6. Solution of periodic Burgers equation with linear polynomials (with post-processing). 
TABLE 1

Convergence rate for the periodic Burgers equation using linear hierarchical basis functions.

\begin{tabular}{|c||c|c||c|c|}
\hline \multicolumn{1}{|c||}{} & \multicolumn{2}{c||}{ without post-processing } & \multicolumn{2}{c|}{ with post-processing } \\
\hline levels & $L^{1}$ error & rate & $L^{1}$ error & rate \\
\hline 8 & $5.775 \mathrm{e}-03$ & - & $5.238 \mathrm{e}-02$ & - \\
\hline 9 & $5.321 \mathrm{e}-04$ & 3.44 & $3.124 \mathrm{e}-03$ & 4.07 \\
\hline 10 & $2.221 \mathrm{e}-05$ & 4.58 & $2.517 \mathrm{e}-05$ & 6.96 \\
\hline 11 & $3.691 \mathrm{e}-06$ & 2.59 & $3.691 \mathrm{e}-06$ & 2.77 \\
\hline 12 & $9.230 \mathrm{e}-07$ & 2.00 & $9.230 \mathrm{e}-07$ & 2.00 \\
\hline 13 & $2.307 \mathrm{e}-07$ & 2.00 & $2.307 \mathrm{e}-07$ & 2.00 \\
\hline 14 & $5.768 \mathrm{e}-08$ & 2.00 & $5.768 \mathrm{e}-08$ & 2.00 \\
\hline
\end{tabular}

error rate with or without post-processing. We have no theoretical justification for these convergence rates, but this is a common failing for conservation laws.

5. Concluding remarks. Initial results for the new method seem promising. We have a stable finite element method which, in some cases, attains quasi-optimal convergence rates in smooth regions. We also have developed a theoretical foundation for understanding why the method works. These results, however, are preliminary. There are potential pitfalls awaiting in more complicated problems, but there is also untapped potential within the framework. Hierarchical bases, for example, should provide a suitable environment for implementing adaptive strategies, both for the grid and the diffusion term.

Acknowledgments. The authors wish to thank Professor Eitan Tadmor and Dr. Curtis Ober for many helpful discussions.

\section{REFERENCES}

[1] R. BANK AND J. Xu, An algorithm for coarsening unstructured meshes, Numer. Math., 73 (1996), pp. 1-36.

[2] W. Bangerth, R. Hartmann, and G. Kanschat, deal. II Differential Equations Analysis Library, Technical Reference, IWR, Heidelberg, http://www.dealii.org.

[3] M. Calhoun-Lopez, Numerical Solutions of Hyperbolic Conservation Laws: Incorporating Multi-Resolution Viscosity Methods into the Finite Element Framework, Ph.D. thesis, Iowa State University, Ames, IA, 2003.

[4] M. Calhoun-Lopez and M. GunzBurger, Implementation and testing of finite element, multiresolution viscosity method for hyperbolic conservation laws, Comp. Methods Appl. Mech. Engrg., submitted.

[5] G. Chen, Q. Du, And E. TADMor, Spectral viscosity approximations to multidimensional scalar conservation laws, Math. Comp., 61 (1993), pp. 629-643.

[6] B. Cockburn, G. Karniadakis, and C.-W. Shu, The development of discontinuous Galerkin methods, in Discontinuous Galerkin Methods Theory, Computation, and Applications, B. Cockburn, G. Karniadakis, and C.-W. Shu, eds., Springer, Berlin, 2000, pp. 3-50.

[7] L. Evans, Weak Convergence Methods for Nonlinear Partial Differential Equations, AMS, Providence, RI, 1990.

[8] L. Evans, Partial Differential Equations, AMS, Providence, RI, 1998.

[9] A. Gelb And E. TADmor, Enhanced spectral viscosity approximations for conservation laws, Appl. Numer. Math., 33 (2000), pp. 3-21.

[10] E. Godlewski and P.-A. Raviart, Numerical Approximation of Hyperbolic Systems of Conservation Laws, Springer, New York, 1996.

[11] S. Gottlieb, C.-W. Shu, and E. Tadmor, Strong stability-preserving high-order time discretization methods, SIAM Rev., 43 (2001), pp. 89-112.

[12] B.-Y. GuO, H.-P. MA, AND E. TADMOR, Spectral vanishing viscosity method for nonlinear conservation laws, SIAM J. Numer. Anal., 39 (2001), pp. 1254-1268.

[13] C. Johnson And A. Szepessy, On the convergence of a finite element method for a nonlinear hyperbolic conservation law, Math. Comp., 49 (1987), pp. 427-444. 
[14] C. Johnson, A. Szepessy, And P. Hansbo, On the convergence of shock-capturing streamline diffusion finite element methods for hyperbolic conservation laws, Math. Comp., 54 (1990), pp. 107-129.

[15] R. Kornhuber and H. Yserentant, Multilevel methods for elliptic problems on domains not resolved by the coarse grid, in Domain Decomposition Methods in Scientific and Engineering Computing, AMS, Providence, RI, 1994, pp. 49-60.

[16] P. Lax, Hyperbolic Systems of Conservation Laws and the Mathematical Theory of Shock Waves, SIAM, Philadelphia, 1973.

[17] R. LeVeque, Numerical Methods for Conservation Laws, Birkhäuser, Basel, 1992.

[18] R. LeVeque, Finite Volume Methods for Hyperbolic Problems, Cambridge University Press, Cambridge, UK, 2002.

[19] Y. MAday, S. M. Ould Kaber, And E. TAdmor, Legendre pseudospectral viscosity method for nonlinear conservation laws, SIAM J. Numer. Anal., 30 (1993), pp. 321-342.

[20] Y. Maday AND E. TADMOR, Analysis of the spectral vanishing viscosity method for periodic conservation laws, SIAM J. Numer. Anal., 26 (1989), pp. 854-870.

[21] F. Murat, L'injection du cône positif de $H^{-1}$ dans $W^{-1, q}$ est compacte pour tout $q<2$, J. Math. Pures Appl. (9), 60 (1981), pp. 309-322.

[22] B. Perthame and E. Tadmor, A kinetic equation with kinetic entropy functions for scalar conservation laws, Comm. Math. Phys., 136 (1991), pp. 501-517.

[23] A. SZEPESSY, Convergence of a shock-capturing streamline diffusion finite element method for a scalar conservation law in two space dimensions, Math. Comp., 53 (1989), pp. 527-545.

[24] E. TADMOR, Convergence of spectral methods for nonlinear conservation laws, SIAM J. Numer. Anal., 26 (1989), pp. 30-44.

[25] E. TAdmor, Total variation and error estimates for spectral viscosity approximations, Math. Comp., 60 (1993), pp. 245-256.

[26] E. TADMOR, Super-viscosity and spectral approximations of nonlinear conservation laws, in Numerical Methods for Fluid Dynamics, Vol. IV, M. J. Baines and K. W. Morton, eds., Oxford University Press, New York, 1993, pp. 69-81.

[27] E. TADMOR, Approximate solutions of nonlinear conservation laws and related equations, in Recent Advances in Partial Differential Equations, AMS, Providence, RI, 1998, pp. 325368.

[28] L. TARTAR, Compensated compactness and applications to partial differential equations, in Nonlinear Analysis and Mechanics: Heriot-Watt Symposium, Vol. IV, Pitman, Boston, 1979, pp. 136-212.

[29] H. Yserentant, On the multilevel splitting of finite element spaces, Numer. Math., 49 (1986), pp. 379-412.

[30] H. Yserentant, Hierarchical bases, in Proceedings of ICIAM 91, R. O'Malley, ed., SIAM, Philadelphia, 1992, pp. 256-276.

[31] O. Zienkiewicz, D. Kelly, J. Gago, and I. BabušKa, Hierarchical finite element approaches, error estimates and adaptive refinement, in The Mathematics of Finite Elements and Applications, Vol. IV, J. Whiteman, ed., Academic Press, New York, 1982, pp. 313-346. 